\title{
In vitro and in silico assessment of antiproliferative activity of new acetamides bearing 1,3,4-oxadiazole and pyrimidine cores via COX inhibition
}

\author{
Belgin SEVER ${ }^{1}$ * (D), Mehlika Dilek ALTINTOP 1 (D), Gülşen AKALIN ÇİFTÇİ 2 (D) \\ 1 Department of Pharmaceutical Chemistry, Faculty of Pharmacy, Anadolu University, 26470 Eskişehir, Turkey. \\ 2 Department of Biochemistry, Faculty of Pharmacy, Anadolu University, 26470 Eskişehir, Turkey. \\ * Corresponding Author. E-mail: belginsever@anadolu.edu.tr (B.S.); Tel. +90-222-335 0580.
}

Received: 01 July 2020 / Revised: 18 August 2020 / Accepted: 27 August 2020

\begin{abstract}
Lung cancer is not only the most commonly diagnosed cancer type but also the leading cause of cancer related deaths throughout the world. The advanced methods for lung cancer therapy have focused on the development of new targeted agents. Cyclooxygenase (COX) is one of the most crucial targets for lung cancer therapy. In the current work, new compounds (1-12) containing 1,3,4-oxadiazole and pyrimidine cores within the acetamide framework were synthesized and evaluated for their cytotoxic effects on A549 human lung adenocarcinoma and NIH/3T3 mouse embryonic fibroblast (healthy) cell lines using MTT assay. Compounds $\mathbf{2}$ and $\mathbf{1 0}$ were defined as the most cytotoxic agents in this series $\left(\mathrm{IC}_{50}<3.9 \mu \mathrm{g} / \mathrm{mL}\right)$ compared to cisplatin $\left(\mathrm{IC}_{50}=26.00 \pm 3.00 \mu \mathrm{g} / \mathrm{mL}\right.$ ) without revealing cytotoxicity to healthy cells. The COX-1 and COX-2 inhibitory profiles of compounds $\mathbf{2}$ and $\mathbf{1 0}$ were also searched for providing a mechanistic insight into their potent antiproliferative effects. Compound 2 inhibited COX-1 and COX-2 dually and significantly (59.52\% and 50.59\%, respectively), whereas compound 10 exhibited no significant COX-1 and COX-2 inhibition. Molecular docking studies indicated that compound 2 showed its COX-1 and COX-2 inhibitory potencies with favourable interactions in the active sites of COX-1 and COX-2. Both in vitro and in silico assays accentuated that potential, orally bioavailable drug-like compound 2 attracted notice for the COX-targeted anti-lung cancer treatment.
\end{abstract}

KEYWORDS: Lung cancer; cyclooxygenase; acetamides; 1,3,4-oxadiazole; pyrimidine.

\section{INTRODUCTION}

Lung cancer (LC), one of the most deadly cancers for both men and women worldwide, is a heterogeneous, invasive and metastatic cancer type. Besides, LC is hardly detectable at early stages (stage I or II) due to its vague symptoms based on its different anatomic location in the bronchial tree [1,2].

LC is mainly divided into two large groups namely, small cell lung carcinoma (SCLC) and non-smallcell lung carcinoma (NSCLC), accounting for $15 \%$ and $85 \%$ of all lung cancers, respectively. Among NSCLC types, adenocarcinoma is the most common type [3].

Patients diagnosed with NSCLC at early stages are generally recommended to undergo surgery unless they have medical contraindications to surgical resection or refuse this treatment option. At advanced stages, radiotherapy and chemotherapy are integrated with the treatment regimen such that the standard cure consists of radiotherapy and chemotherapy using either cisplatin or carboplatin and a second drug. However, in recent years standart chemotherapy has altered to targeted therapy that regulate the components involved in the downstream of signaling pathways underlying tumor growth and progression [4-7].

Cyclooxygenase (COX) is a membrane-bound enzyme, which catalyzes the first two steps of the pathway forming prostaglandins (PGs) and thromboxane (TX) $\mathrm{A}_{2}$. There are mainly two isoforms of COX: COX-1 is constitutively expressed in most tissues playing important roles such as the synthesis of cytoprotective prostaglandins in the gastrointestinal tract and TXA 2 in blood platelets. On the other hand, COX-2 is normally undetectable in most tissues but it is easily expressed by a variety of stimuli associated with inflammatory response [8-12].

Numerous studies showed that COX-2 has been overexpressed in several different cancers including particularly colon cancer and other cancer forms such as lung, breast, head and neck, prostate, bone marrow, pancreatic and liver cancers. In preclinical models, it was demonstrated that increased levels of COX-2

How to cite this article: Sever B, Altıntop MD, Akalın Çiftçi G. In vitro and in silico assessment of antiproliferative activity of new acetamides bearing 1,3,4-oxadiazole and pyrimidine cores via COX inhibition. J Res Pharm. 2020; 24(5): 656-669. 
repressed apoptosis, promoted tumor-cell invasion and enhanced angiogenesis in tumor cells, whereas the relevant mechanisms have not all been elucidated. Besides, higher COX-2 levels have been reported to overexpress in almost all NSCLC precursor lesions leading to a worse overall survival rate [13-21]. Although COX-1 has been considered not to be involved in carcinogenesis due to its homeostatic cell and tissue functions, there are many reports showing the connection between the increased levels of COX-1 and cancer pathophysiology [22-25].

1,3,4-Oxadiazoles, bioisosteres of amides and esters, have attracted a great deal of interest with their ability of forming hydrogen bonding in receptor/enzyme site and their lipophilicity affecting the transmembrane transport explicitly [26, 27]. Zibotentan (ZD4054) containing a 1,3,4-oxadiazole ring (Figure 1) was found to be beneficial for patient survival in a phase II study about metastatic castration-resistant prostate cancer. Although ZD4054 failed a phase III clinical trial for prostate cancer, it has been a lead compound for the development of 1,3,4-oxadiazole-based new anticancer agents [28].<smiles>COc1nc(C)cnc1NS(=O)(=O)c1cccnc1-c1ccc(-c2nnco2)cc1</smiles>

Figure 1. Zibotentan.

Since pyrimidine nucleotides are essential for the synthesis of deoxyribonucleic acid (DNA) and ribonucleic acid (RNA), pyrimidine bearing compounds have been widely investigated for diverse activities including anticancer activity in the regular literature and patents field [29-32]. Brigatinib (Figure 2) with bisanilino-pyrimidine structure is a novel anaplastic lymphoma kinase (ALK) inhibitor, which was found effective in the treatment of NSCLC [33]. Abemaciclib, imatinib, nilotinib, nimustine, osimertinib, pazopanib and rociletinib (Figure 2) are another pyrimidine-based anticancer agents [34,35]. Recent studies also draw attention to pyrimidine ring to be a useful template for the synthesis of new selective COX-2 inhibitors [3638].<smiles>COc1cc(N2CCC(N3CCN(C)CC3)CC2)ccc1Nc1ncc(Cl)c(Nc2ccccc2P(C)(C)=O)n1</smiles><smiles>Cc1ncc(CNC(=O)N(CCCl)N=O)c(N)n1</smiles><smiles></smiles>

Abemaciclib<smiles>Cc1cn(-c2cc(NC(=O)c3ccc(C)c(Nc4nccc(-c5cccnc5)n4)c3)cc(C(F)(F)F)c2)cn1</smiles><smiles>C=CC(=O)Nc1cccc(Nc2nc(Nc3ccc(N4CCN(C(C)=O)CC4)cc3OC)ncc2C(F)(F)F)c1</smiles><smiles></smiles>

Nilotinib

Osimertinib

Figure 2. Pyrimidine-based anticancer agents. 
In the current work, our efforts were devoted to synthesize new acetamides carrying 1,3,4-oxadiazole and pyrimidine cores (1-12) and further investigate their anti-lung cancer effects on A549 human lung adenocarcinoma cell line via COX-1 and COX-2 inhibition. The selectivity of their cytotoxic effects was also determined using NIH/3T3 mouse embryonic fibroblast (healthy) cell line. Molecular docking studies were also performed in the active sites of COX-1 and COX-2 to shed light on the in vitro assays by exploring the possible interactions of the most effective agents in this series. Moreover, some physicochemical properties and the bioavailability of all compounds were also in silico predicted.

\section{RESULTS AND DISCUSSION}

A facile synthetic route was rationally designed as described in Figure 3 to obtain a new series of oxadiazoles (1-12) efficiently. In brief, 2-[(4-(4-chlorophenyl)pyrimidin-2-yl)thio)]acetohydrazide was synthesized via the reaction of hydrazine hydrate with ethyl 2-[(4-(4-chlorophenyl)pyrimidin-2-yl)thio]acetate, which was obtained via the reaction of 4-(4-chlorophenyl)pyrimidine-2-thiol with ethyl chloroacetate in the presence of potassium carbonate. The ring closure reaction of the hydrazide with carbon disulfide in the presence of potassium hydroxide led to the formation of the oxadiazole scaffold carrying a thione group in the $2^{\text {nd }}$ position found in the title compounds. Finally, 5-[((4-(4-chlorophenyl)pyrimidin-2-yl)thio)methyl]-1,3,4oxadiazole-2 $(3 H)$-thione was reacted with 2-chloro- $N$-(aryl)acetamide/4-(chloroacetyl)morpholine in the presence of potassium carbonate to afford compounds 1-12. Their structures were verified by different spectroscopic techniques, namely Infrared (IR), ${ }^{1} \mathrm{H}$ Nuclear Magnetic Resonance (NMR), ${ }^{13} \mathrm{C}$ NMR and High Resolution Mass Spectrometry (HRMS).
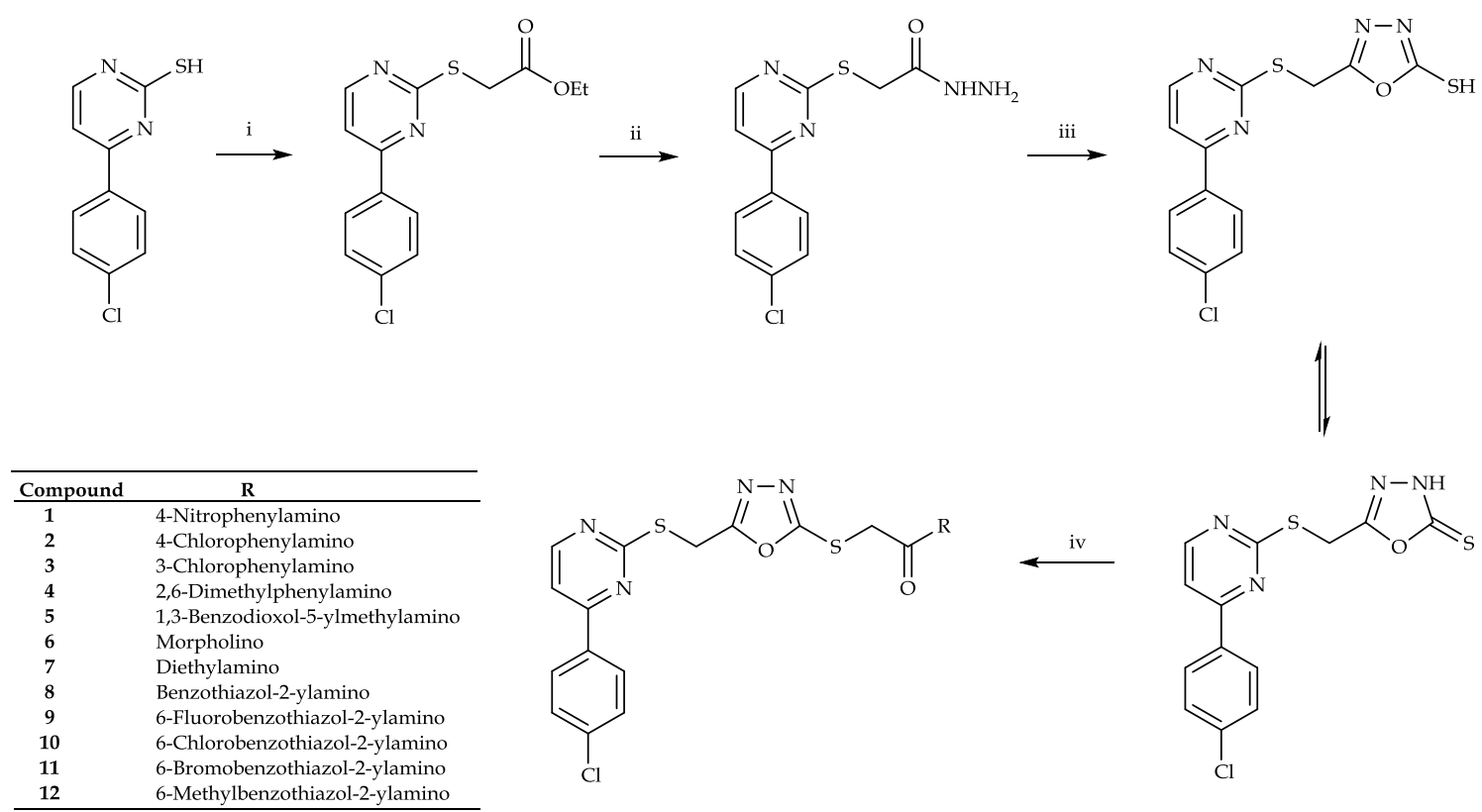

Figure 3. The synthetic route for the preparation of compounds 1-12. Reagents and conditions: (i) $\mathrm{ClCH}_{2} \mathrm{COOC}_{2} \mathrm{H}_{5}, \mathrm{~K}_{2} \mathrm{CO}_{3}$, acetone, reflux, $12 \mathrm{~h}$; (ii) $\mathrm{NH}_{2} \mathrm{NH}_{2} . \mathrm{H}_{2} \mathrm{O}$, ethanol, rt, 5 h; (iii) $\mathrm{CS}_{2}, \mathrm{KOH}$, ethanol, reflux, $8 \mathrm{~h}$; (iv) 2-Chloro- $N$-(aryl)acetamide/4-(chloroacetyl)morpholine, $\mathrm{K}_{2} \mathrm{CO}_{3}$, acetone, $\mathrm{rt}, 8 \mathrm{~h}$.

In the IR spectra of compounds 1-12, the $\mathrm{C}=\mathrm{O}$ stretching vibration resulted in the formation of the characteristic amide I band at 1681.93-1641.42 $\mathrm{cm}^{-1}$. The N-H stretching vibrations belonging to the secondary amide group gave rise to the bands in the region 3292.49-3172.90 $\mathrm{cm}^{-1}$. Aromatic and aliphatic C-H stretching bands were observed at 3149.76-3010.88 $\mathrm{cm}^{-1}$ and 2991.59-2837.29 $\mathrm{cm}^{-1}$, respectively. N-H bending, $\mathrm{C}=\mathrm{N}$ and $\mathrm{C}=\mathrm{C}$ stretching bands appeared between $1645.28 \mathrm{~cm}^{-1}$ and $1456.26 \mathrm{~cm}^{-1}$.

In the ${ }^{1} \mathrm{H}$ NMR spectra of compounds 1-12, the both $\mathrm{S}-\mathrm{CH}_{2}$ protons appeared as singlet at $4.05-4.75 \mathrm{ppm}$. Besides, the protons of (1,3-benzodioxol-5-ylmethyl) moiety belonging to compound $\mathbf{5}$ were observed as singlet at $4.73 \mathrm{ppm}\left(\mathrm{NH}-\mathrm{CH}_{2}\right)$ and $5.96 \mathrm{ppm}\left(\mathrm{O}-\mathrm{CH}_{2}-\mathrm{O}\right)$. The methylene protons of morpholine ring of compound 6 resonated as triplet at $3.44 \mathrm{ppm}\left(\mathrm{H}_{2} \mathrm{C}-\mathrm{N}-\mathrm{CH}_{2}, J=5.25,4.35,9.60 \mathrm{~Hz}, 4 \mathrm{H}\right)$ and at $3.54 \mathrm{ppm}(J=4.45$, $9.40 \mathrm{~Hz}, 2 \mathrm{H})$ and $3.58 \mathrm{ppm}(J=4.40,4.70,9.10 \mathrm{~Hz}, 2 \mathrm{H})\left(\mathrm{H}_{2} \mathrm{C}-\mathrm{O}-\mathrm{CH}_{2}\right)$, whereas the methylene protons of $N, N-$ diethyl group of compound 7 resonated as quartet at $3.26 \mathrm{ppm}(J=7.15,14.25 \mathrm{~Hz}, 2 \mathrm{H})$ and $3.31 \mathrm{ppm}(J=7.10$, 
14.25 Hz, 2H). The NH protons of compounds 1-12, except for compounds 6 and 7, were determined at 10.38$10.83 \mathrm{ppm}$. The methyl protons were detected as singlet at $1.98 \mathrm{ppm}$ and $2.07 \mathrm{ppm}$ for compound $\mathbf{4}$, at 2.39 ppm for compound 12 and as triplet at $1.00 \mathrm{ppm}(J=7.05,7.10,14.15 \mathrm{~Hz}, 3 \mathrm{H})$ and $1.11 \mathrm{ppm}(J=7.10,14.20 \mathrm{~Hz}$, 3H) for compound 7.

In the ${ }^{13} \mathrm{C}$ NMR spectra of compounds 1-12, the both $\mathrm{S}-\mathrm{CH}_{2}$ carbons appeared at 24.57-36.85 ppm. The carbons of (1,3-benzodioxol-5-ylmethyl) moiety of compound 5 resonated at $42.89 \mathrm{ppm}\left(\mathrm{NH}-\mathrm{CH}_{2}\right)$ and 101.40 ppm $\left(\mathrm{O}-\mathrm{CH}_{2}-\mathrm{O}\right)$, whereas the methylene carbons of morpholine ring of compound $\mathbf{6}$ were determined at 42.50 ppm, $46.19 \mathrm{ppm}\left(\mathrm{H}_{2} \mathrm{C}-\mathrm{N}-\mathrm{CH}_{2}\right)$ and $66.31 \mathrm{ppm}\left(\mathrm{H}_{2} \mathrm{C}-\mathrm{O}-\mathrm{CH}_{2}\right)$. The carbons of $\mathrm{N}, \mathrm{N}$-diethyl group of compound 7 were detected at $13.29 \mathrm{ppm}$ and $14.32 \mathrm{ppm}\left(\mathrm{CH}_{3}\right)$ and $42.31 \mathrm{ppm}\left(2 \mathrm{CH}_{2}\right)$. The methyl carbons were observed at $17.65 \mathrm{ppm}$ and $18.52 \mathrm{ppm}$ for compound 4, whereas the methyl carbon of compound $\mathbf{1 2}$ appeared at 21.44 $\mathrm{ppm}$. The characteristic carbonyl carbons of compounds 1-12 gave rise to the peaks at 165.54-171.07 ppm. All the aromatic protons and carbons of compounds 1-12 were consistent with the proposed structures of the compounds. Finally, the HRMS data of all compounds were coherent with their molecular formulas.

Compounds 1-12 were screened for their cytotoxic effects on A549 human lung adenocarcinoma and $\mathrm{NIH} / 3 \mathrm{~T} 3$ mouse embryonic fibroblast (healthy) cell lines (Table 1) using MTT test. Compounds 2, $\mathbf{4}$ and $\mathbf{1 0}$ were identified as the most cytotoxic agents in this series against A549 cells with IC $_{50}$ values lower than 3.9 $\mu \mathrm{g} / \mathrm{mL}$ when compared with cisplatin $\left(\mathrm{IC}_{50}=26.00 \pm 3.00 \mu \mathrm{g} / \mathrm{mL}\right)$. Compounds 2 and 10 revealed no cytotoxicity against $\mathrm{NIH} / 3 \mathrm{~T} 3$ cells at their effective concentrations $\left(\mathrm{IC}_{50}=41.00 \pm 9.54 \mu \mathrm{g} / \mathrm{mL}\right.$ and $20.00 \pm 2.00$ $\mu \mathrm{g} / \mathrm{mL}$, respectively), whereas compound 4 displayed cytotoxic activity against healthy cells with an $\mathrm{IC}_{50}$ value of $<3.9 \mu \mathrm{g} / \mathrm{mL}$. According to the results, 4-chlorophenylamino and 6-chlorobenzothiazol-2-ylamino moieties enhanced the antiproliferative effects of compounds 2 and 10, respectively. However, 3chlorophenylamino substitution led to a reduction in antiproliferative activity of compound 3. The 4nitrophenylamino substituted compound 1 exhibited moderate antiproliferative activity with an $\mathrm{IC}_{50}$ value of $39.67 \pm 5.03 \mu \mathrm{g} / \mathrm{mL}$ exerting no cytotoxicity to $\mathrm{NIH} / 3 \mathrm{~T} 3$ cells $\left(\mathrm{IC}_{50}=380.00 \pm 103.90 \mu \mathrm{g} / \mathrm{mL}\right.$ ) (Table 1 ).

Table 1. $\mathrm{IC}_{50}$ values of the compounds for A549 and NIH/3T3 cells after $24 \mathrm{~h}$.

\begin{tabular}{ccc}
\hline \multirow{2}{*}{ Compound } & \multicolumn{2}{c}{ IC $_{50}(\mu \mathrm{g} / \mathrm{mL})$} \\
\cline { 2 - 3 } & A549 Cell Line & NIH/3T3 Cell Line \\
\hline $\mathbf{1}$ & $39.67 \pm 5.03$ & $380.00 \pm 103.90$ \\
$\mathbf{2}$ & $<3.90$ & $41.00 \pm 9.54$ \\
$\mathbf{3}$ & $191.67 \pm 16.07$ & $<3.90$ \\
$\mathbf{4}$ & $<3.90$ & $<3.90$ \\
$\mathbf{5}$ & $402.50 \pm 67.18$ & $>500.00$ \\
$\mathbf{6}$ & $48.50 \pm 4.95$ & $34.00 \pm 9.64$ \\
$\mathbf{7}$ & $193.33 \pm 5.28$ & $12.50 \pm 0.71$ \\
$\mathbf{8}$ & $>500.00$ & $<3.90$ \\
$\mathbf{9}$ & $400.00 \pm 10.00$ & $120.00 \pm 26.46$ \\
$\mathbf{1 0}$ & $<3.90$ & $20.00 \pm 2.00$ \\
$\mathbf{1 1}$ & $200.00 \pm 17.02$ & $86.50 \pm 12.20$ \\
$\mathbf{1 2}$ & $410.00 \pm 10.00$ & $42.30 \pm 2.52$ \\
\hline Cisplatin & $18.33 \pm 0.94$ & $\mathrm{NT}$ \\
\hline
\end{tabular}

NT: Not Tested.

As compounds $\mathbf{2}$ and $\mathbf{1 0}$ were found as the most potent antiproliferative agents against A549 cells, they were further investigated for their COX-1 and COX-2 inhibitory potencies. The results indicated that compound 2 significantly inhibited both COX-1 and COX-2 (59.52\% and 50.59\%, respectively) compared to cisplatin $(24.41 \%$ and $43.07 \%$, respectively). This outcome remarks the importance of 4 -chlorophenylamino substitution for COX inhibition. However, compound $\mathbf{1 0}$ showed no significant inhibition for both COX-1 and COX-2 (3.79\% and $12.14 \%$, respectively). 
Table 2. The COX activities $(\mathrm{U} / \mathrm{mL})$ and the inhibitory effects of compounds $\mathbf{2 ,} 10$ and cisplatin (at $\mathrm{IC}_{50}$ values) on COXs in A549 cells after $24 \mathrm{~h}$ incubation.

\begin{tabular}{ccccc}
\hline & $\begin{array}{c}\text { COX-1 activity } \\
(\mathbf{U} / \mathbf{m L})\end{array}$ & $\begin{array}{c}\text { COX-2 activity } \\
\mathbf{( U / m L )}\end{array}$ & $\begin{array}{c}\text { COX-1 } \\
\text { inhibition } \%\end{array}$ & $\begin{array}{c}\text { COX-2 } \\
\text { inhibition } \%\end{array}$ \\
\hline Control & 4.04 & 4.16 & $\mathrm{NT}$ & $\mathrm{NT}$ \\
Compound 2 & 1.63 & 2.05 & 59.52 & 50.59 \\
Compound 10 & 3.87 & 3.65 & 3.79 & 12.14 \\
\hline Cisplatin & 3.10 & 2.40 & 24.41 & 43.07 \\
\hline COX-1 & 9.62 & $\mathrm{NT}$ & $\mathrm{NT}$ & $\mathrm{NT}$ \\
standard & & & & \\
\hline
\end{tabular}

NT: Not Tested.

Molecular docking studies were conducted for compound 2 to sort out the feasible interactions in the active sites of both COX-1 and COX-2 (PDB IDs: 3KK6 and 5JW1, respectively). The 4-chlorophenyl group at the $4^{\text {th }}$ position of the pyrimidine ring forged a $\Pi-\Pi$ stacking interaction with Tyr355, whereas pyrimidine scaffold formed a hydrogen bonding with Ser516 in the active site of COX-1 (Figure 4). Both the 1,3,4oxadiazole and pyrimidine cores were engaged in п-п interactions with Arg121 and the 1,3,4-oxadiazole core also presented a $\Pi-\Pi$ stacking interaction with Tyr356. Besides, compound 2 formed a hydrogen bonding with Tyr116 through its acetamido group in the active site of COX-2 (Figure 5). The docking scores and glide scores of compound 2 were found as $-7.65 \mathrm{kcal} / \mathrm{mol}$ for COX-1 and $-7.70 \mathrm{kcal} / \mathrm{mol}$ for COX-2 indicating the high affinity to both enzymes.

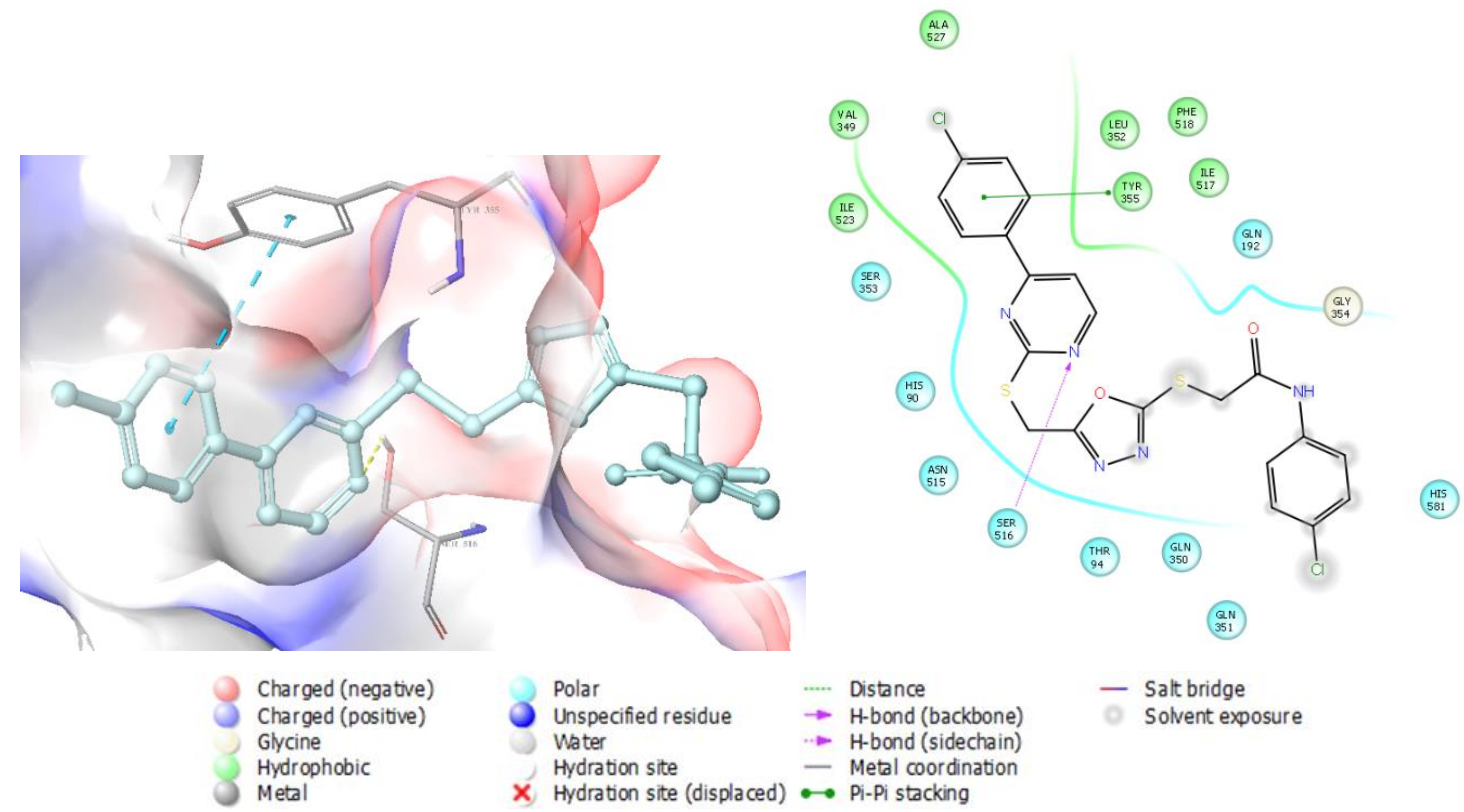

Figure 4. Docking pose and interactions of compound 2 in the active site of COX-1 (Blue dashes: $\Pi-\Pi$ stacking interaction, yellow dashes: hydrogen bonding).

Some Absorption, Distribution, Metabolism and Excretion (ADME) properties including predicted octanol/water partition coefficient (QPlogPo/w), brain/blood partition coefficient (QPlogBB), human serum albumin binding (QPlogKhsa) and predicted human oral absorption were in silico ascertained. The results given in Table 3 were within the acceptable range based on the specified parameters making these compounds suitable drug candidates excluding compounds $\mathbf{1}$ and $\mathbf{8}$ as their predicted human oral absorption rates were found below $80.00 \%$. 


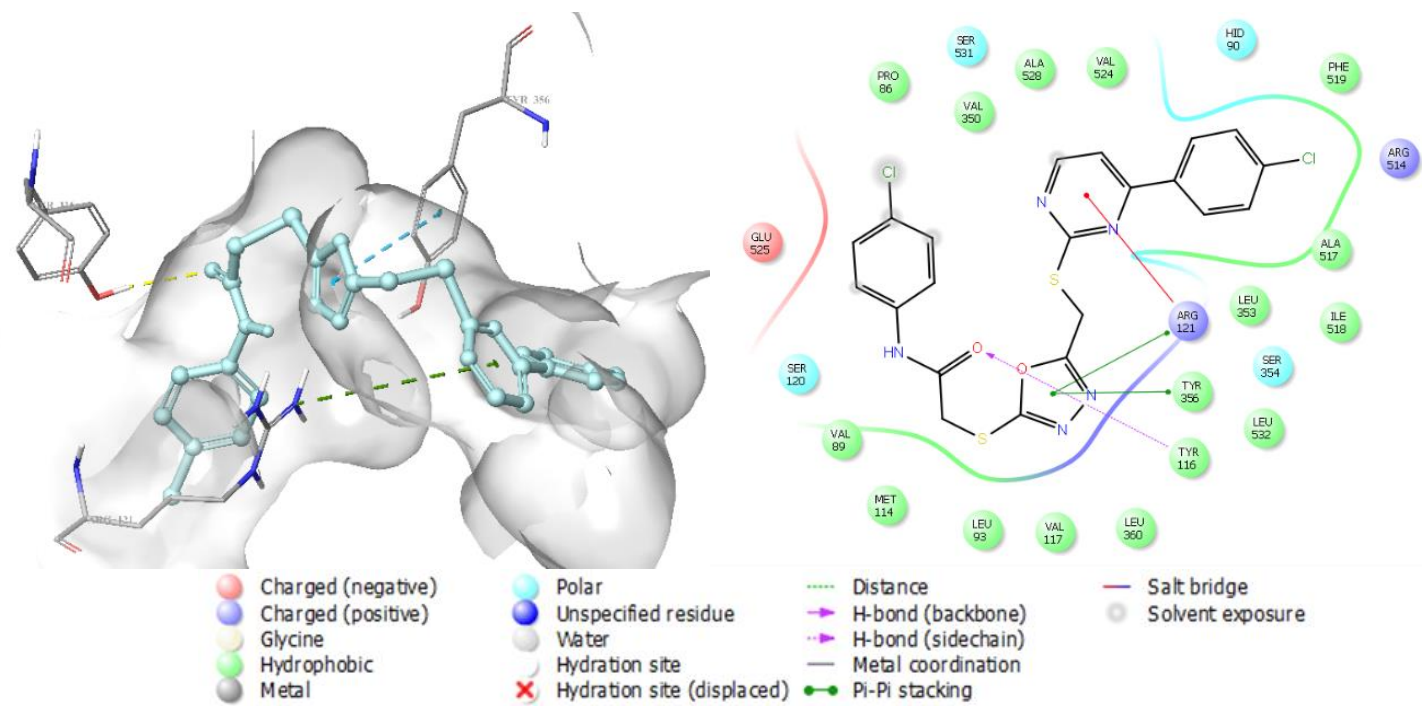

Figure 5. Docking pose and interactions of compound 2 in the active site of COX-2 (Blue dashes: $\Pi-\Pi$ stacking interaction, yellow dashes: hydrogen bonding).

Table 3. Predicted ADME properties of compounds 1-12.

\begin{tabular}{ccccc}
\hline Compound & QPlogPo/w $^{*}$ & QPlogBB $^{*}$ & QPlogKhsa $^{*}$ & Human oral absorption $^{*}{ }^{*}$ \\
\hline $\mathbf{1}$ & 4.47 & -2.31 & 0.54 & 73.78 \\
$\mathbf{2}$ & 5.80 & -0.80 & 0.73 & 86.66 \\
$\mathbf{3}$ & 5.80 & -0.80 & 0.73 & 86.64 \\
$\mathbf{4}$ & 5.86 & -0.99 & 0.91 & 100.00 \\
$\mathbf{5}$ & 4.53 & -0.97 & 0.18 & 87.85 \\
$\mathbf{6}$ & 2.94 & -0.88 & -0.57 & 90.85 \\
$\mathbf{7}$ & 3.97 & -0.85 & -0.16 & 100.00 \\
$\mathbf{8}$ & 5.21 & -1.22 & 0.55 & 78.78 \\
$\mathbf{9}$ & 5.45 & -1.12 & 0.60 & 80.15 \\
$\mathbf{1 0}$ & 5.70 & -1.07 & 0.67 & 81.67 \\
$\mathbf{1 1}$ & 5.78 & -1.06 & 0.69 & 82.16 \\
$\mathbf{1 2}$ & 5.52 & -1.26 & 0.71 & 80.58 \\
\hline
\end{tabular}

* QPlogPo/w: Predicted octanol/water partition coefficient (-2.00-6.5), QPlogBB: brain/blood partition coefficient (-3-1.2), QPlogKhsa: binding to human serum albumin (-1.5-1.5), Percent human oral absorption: human oral absorption on $0-100 \%$ scale $(>80 \%$ is high, $<25 \%$ is poor).

\section{CONCLUSION}

In the current work, new acetamides clubbed with 1,3,4-oxadiazole and pyrimidine skeletons (1-12) were obtained via efficient and concise synthetic methods. These compounds were screened for their anti-lung cancer effects on A549 cells along with their cytotoxic effects on NIH/3T3 (healthy) cells. Compounds 2 and 10 displayed potent and selective antiproliferative activity against A549 cell line compared to cisplatin. These two compounds were further investigated to find out whether they inhibited COX-1 and COX-2 or not. Compound 2 exhibited significant COX-1 and COX-2 inhibitory activity in A549 cells. Molecular docking approaches in this work also indicated that reasonable interactions of compound $\mathbf{2}$ in the active sites of COX1 and COX-2 supported in vitro studies. Some physicochemical parameters were also in silico predicted revealing that this compound was a highly potent orally bioavailable drug-like molecule. 


\section{MATERIALS AND METHODS}

\subsection{Chemistry}

All reagents purchased from commercial suppliers were used without further purification. The Electrothermal IA9200 digital melting point apparatus (Staffordshire, UK) was used to determine the melting points (M.p.) of the compounds. IR spectra were recorded on an IRPrestige-21 Fourier Transform Infrared spectrophotometer (Shimadzu, Tokyo, Japan). ${ }^{1} \mathrm{H}$ NMR and ${ }^{13} \mathrm{C}$ NMR spectra were recorded on a Bruker 500 $\mathrm{MHz}$ spectrometer (Bruker, Billerica, MA, USA). HRMS spectra were recorded on a Shimadzu LCMS-IT-TOF system (Shimadzu, Kyoto, Japan).

\subsubsection{General procedure for the synthesis of the compounds}

Ethyl 2-[(4-(4-chlorophenyl)pyrimidin-2-yl)thio]acetate

A mixture of 4-(4-chlorophenyl)pyrimidine-2-thiol $(0.067 \mathrm{~mol})$ and ethyl chloroacetate $(0.067 \mathrm{~mol})$ in the presence of potassium carbonate $(0.067 \mathrm{~mol})$ in acetone was refluxed for $12 \mathrm{~h}$. The reaction mixture was cooled, filtered and washed with water [39].

\section{2-[(4-(4-Chlorophenyl)pyrimidin-2-yl)thio)]acetohydrazide}

A mixture of the ester $(0.06 \mathrm{~mol})$ and hydrazine hydrate $(0.12 \mathrm{~mol})$ in ethanol was stirred at room temperature for $5 \mathrm{~h}$. The reaction mixture was filtered and washed with ethanol [39].

\section{5-[((4-(4-Chlorophenyl)pyrimidin-2-yl)thio)methyl]-1,3,4-oxadiazole-2(3H)-thione}

A mixture of the hydrazide $(0.025 \mathrm{~mol})$ and carbon disulfide $(0.03 \mathrm{~mol})$ in the presence of potassium hydroxide $(0.025 \mathrm{~mol})$ in ethanol $(50 \mathrm{~mL})$ was refluxed for $8 \mathrm{~h}$. The solution was cooled and acidified with hydrochloric acid solution. The solid was filtered off, washed with water and dried. The product was crystallized from ethanol.

\section{N-(Alkyl/Aryl)-2-[[5-[((4-(4-chlorophenyl)pyrimidin-2-yl)thio)methyl]-1,3,4-oxadiazol-2-yl]thio]acetamides (1-12)}

A mixture of 5-[((4-(4-chlorophenyl)pyrimidin-2-yl)thio)methyl]-1,3,4-oxadiazole-2(3H)-thione $(0.0015$ $\mathrm{mol})$ and 2-chloro- $\mathrm{N}$-(aryl)acetamide/4-(chloroacetyl)morpholine $(0.0015 \mathrm{~mol})$ in acetone $(25 \mathrm{~mL})$ was stirred at room temperature for $8 \mathrm{~h}$ in the presence of potassium carbonate $(0.0015 \mathrm{~mol})$. The solvent was evaporated under reduced pressure. The residue was washed with water and crystallized from ethanol.

2-[(5-((4-(4-Chlorophenyl)pyrimidin-2-yl)thio)methyl)-1,3,4-oxadiazol-2-yl)thio]-N-(4-nitrophenyl)acetamide (1)

M.p. 273-274 ${ }^{\circ} \mathrm{C}$. Yield $46 \%$. IR $v_{\max }\left(\mathrm{cm}^{-1}\right)$ : 3211.48 (N-H stretching), 3088.03, 3049.46 (Aromatic C-H stretching), 2974.23, 2837.29 (Aliphatic C-H stretching), 1672.28 (C=O stretching), 1595.13, 1556.55, 1537.27, $1506.41,1489.05\left(\mathrm{~N}-\mathrm{H}\right.$ bending, $\mathrm{NO}_{2}, \mathrm{C}=\mathrm{N}$ and $\mathrm{C}=\mathrm{C}$ stretching), 1425.40, 1396.46, 1330.88, 1259.52, 1199.72, 1176.58, 1111.00, 1091.71, $1012.63\left(\mathrm{C}-\mathrm{H}\right.$ bending, $\mathrm{NO}_{2}, \mathrm{C}-\mathrm{N}, \mathrm{C}-\mathrm{O}$ stretching and aromatic $\mathrm{C}-\mathrm{H}$ in plane bending), 852.54, 817.82, 773.46, 750.31, 731.02, 690.52, 667.37 (Aromatic C-H out of plane bending and C-S stretching). ${ }^{1} \mathrm{H}$ NMR (500 MHz, DMSO- $\left.d_{6}\right): 4.08$ (s, $2 \mathrm{H}, \mathrm{S}^{\left.-\mathrm{CH}_{2} \mathrm{CO}\right)}, 4.64\left(\mathrm{~s}, 2 \mathrm{H}, \mathrm{S}-\mathrm{CH}_{2}\right), 7.43-7.56(\mathrm{~m}, 2 \mathrm{H}, \mathrm{Ar}-\mathrm{H})$, 7.71-7.84 (m, 5H, Ar-H), 8.00-8.17 (m, 2H, Ar-H), 8.67 (p, J= 6.60, 7.75, $11.30 \mathrm{~Hz}, 1 \mathrm{H}, \mathrm{Ar}-\mathrm{H}), 10.83(\mathrm{~s}, 1 \mathrm{H}, \mathrm{N}-\mathrm{H})$. ${ }^{13} \mathrm{C}$ NMR (125 MHz, DMSO-d 6 ): $33.56\left(\mathrm{CH}_{2}, \mathrm{~S}-\mathrm{CH}_{2}\right), 36.70\left(\mathrm{CH}_{2}, \mathrm{~S}-\mathrm{CH}_{2} \mathrm{CO}\right), 112.82(\mathrm{CH}, \mathrm{C}-5$, pyrimidine), 119.28 (2CH, C-2 and C-6, 4-nitrophenyl), 124.19 (CH, C-3, 4-nitrophenyl), 125.32 (CH, C-5, 4-nitrophenyl), 129.29 (CH, C-2, 4-chlorophenyl), 129.41 (CH, C-6, 4-chlorophenyl), 129.48 (CH, C-3, 4-chlorophenyl), 129.51 (CH, C-5, 4-chlorophenyl), 134.81 (C, C-1, 4-chlorophenyl), 136.83 (C, C-4, 4-chlorophenyl), 142.66 (C, C-4, 4nitrophenyl), 145.55 (C, C-1, 4-nitrophenyl), 155.93 (CH, C-6, pyrimidine), 159.15 (C, C-2, oxadiazole), 162.12 (C, C-5, oxadiazole), 164.52 (C, C-4, pyrimidine), 168.90 (C, C=O), 171.38 (C, C-2, pyrimidine). HRMS (ESI) $(\mathrm{m} / \mathrm{z})[\mathrm{M}+\mathrm{H}]^{+}$calcd. for $\mathrm{C}_{21} \mathrm{H}_{15} \mathrm{ClN}_{6} \mathrm{O}_{4} \mathrm{~S}_{2}: 515.0357$, found: 515.0361.

\section{2-[(5-(((4-(4-Chlorophenyl)pyrimidin-2-yl)thio)methyl)-1,3,4-oxadiazol-2-yl)thio]-N-(4-chlorophenyl)acetamide (2)}

M.p. 248-249 ${ }^{\circ} \mathrm{C}$. Yield 74\%. IR $\mathrm{v}_{\max }\left(\mathrm{cm}^{-1}\right): 3230.77,3182.55$ (N-H stretching), 3118.90, 3062.96 (Aromatic C-H stretching), 2991.59, 2935.66 (Aliphatic C-H stretching), 1674.21 (C=O stretching), 1614.42, 1597.06, $1560.41,1535.34,1490.97$ ( $\mathrm{N}-\mathrm{H}$ bending, $\mathrm{C}=\mathrm{N}$ and $\mathrm{C}=\mathrm{C}$ stretching), 1425.40, 1396.46, 1334.74, 1244.09, 1232.51, 1201.65, 1178.51, 1091.71, 1012.63 (C-H bending, C-N, C-O stretching and aromatic C-H in plane bending), 977.91, 893.04, 812.03, 773.46, 731.02, 702.09, 667.37 (Aromatic C-H out of plane bending and C-S stretching). ${ }^{1} \mathrm{H}$ NMR (500 MHz, DMSO-d $\left.d_{6}\right): 4.05$ (s, 2H, S-CH $\mathrm{CH}_{2} \mathrm{CO}$ ), $4.38\left(\mathrm{~s}, 2 \mathrm{H}, \mathrm{S}-\mathrm{CH}_{2}\right), 7.26$ (d, J= $\left.8.85 \mathrm{~Hz}, 1 \mathrm{H}, \mathrm{Ar}-\mathrm{H}\right), 7.34$ 
$(\mathrm{d}, J=8.65 \mathrm{~Hz}, 3 \mathrm{H}, \mathrm{Ar}-\mathrm{H}), 7.43(\mathrm{~s}, 1 \mathrm{H}, \mathrm{Ar}-\mathrm{H}), 7.55-7.58(\mathrm{~m}, 1 \mathrm{H}, \mathrm{Ar}-\mathrm{H}), 7.74-7.80(\mathrm{~m}, 1 \mathrm{H}, \mathrm{Ar}-\mathrm{H}), 8.21$ (d, J= 8.55 $\mathrm{Hz}, 2 \mathrm{H}, \mathrm{Ar}-\mathrm{H}), 8.62-8.69(\mathrm{~m}, 1 \mathrm{H}, \mathrm{Ar}-\mathrm{H}), 10.38(\mathrm{~s}, 1 \mathrm{H}, \mathrm{N}-\mathrm{H}) .{ }^{13} \mathrm{C}$ NMR $\left(125 \mathrm{MHz}, \mathrm{DMSO}-d_{6}\right): 33.09\left(\mathrm{CH}_{2}, \mathrm{~S}_{-} \mathrm{CH}_{2}\right)$, $36.85\left(\mathrm{CH}_{2}, \mathrm{~S}-\mathrm{CH}_{2} \mathrm{CO}\right), 112.74(\mathrm{CH}, \mathrm{C}-5$, pyrimidine), $121.16(2 \mathrm{CH}, \mathrm{C}-2$ and $\mathrm{C}-6,4$-chlorophenyl), $129.09(\mathrm{CH}$, C-3, 4-chlorophenyl), 129.17 ( $\mathrm{CH}, \mathrm{C}-5$, 4-chlorophenyl), 129.29 (CH, C-2, 4-chlorophenyl), 129.34 (CH, C-6, 4chlorophenyl), 130.32 ( $\mathrm{CH}, \mathrm{C}-3$, 4-chlorophenyl), 130.50 (CH, C-5, 4-chlorophenyl), 133.28 (C, C-1, 4chlorophenyl), 134.59 (C, C-4, 4-chlorophenyl), 134.97 (C, C-1, 4-chlorophenyl), 136.67 (C, C-4, 4-chlorophenyl), 155.85 ( $\mathrm{CH}, \mathrm{C}-6$, pyrimidine), 159.05 (C, C-2, oxadiazole), 162.23 (C, C-5, oxadiazole), 165.18 (C, C-4, pyrimidine), $168.29(\mathrm{C}, \mathrm{C}=\mathrm{O}), 171.72\left(\mathrm{C}, \mathrm{C}-2\right.$, pyrimidine). HRMS (ESI) $(\mathrm{m} / \mathrm{z})[\mathrm{M}+\mathrm{H}]^{+}$calcd. for $\mathrm{C}_{21} \mathrm{H}_{15} \mathrm{Cl}_{2} \mathrm{~N}_{5} \mathrm{O}_{2} \mathrm{~S}_{2}: 504.0117$, found: 504.0092 .

\section{2-[(5-(((4-(4-Chlorophenyl)pyrimidin-2-yl)thio)methyl)-1,3,4-oxadiazol-2-yl)thio]-N-(3-chlorophenyl)acetamide (3)}

M.p. $271-272{ }^{\circ} \mathrm{C}$. Yield $64 \%$. IR $v_{\max }\left(\mathrm{cm}^{-1}\right): 3211.48$ (N-H stretching), 3062.96 (Aromatic C-H stretching), 2980.02, 2929.87 (Aliphatic C-H stretching), 1681.93 (C=O stretching), 1645.28, 1593.20, 1558.48, 1537.27, 1477.47 (N-H bending, $\mathrm{C}=\mathrm{N}$ and $\mathrm{C}=\mathrm{C}$ stretching), 1423.47, 1379.10, 1346.31, 1246.02, 1199.72, 1182.36, 1089.78, 1012.63 (C-H bending, C-N, C-O stretching and aromatic C-H in plane bending), 975.98, 881.47, 819.75, 806.25, 777.31, 731.02, 680.87 (Aromatic C-H out of plane bending and C-S stretching). ${ }^{1} \mathrm{H}$ NMR (500 MHz, DMSO- $d_{6}$ ): $4.17\left(\mathrm{~s}, 2 \mathrm{H}, \mathrm{S}-\mathrm{CH}_{2} \mathrm{CO}\right), 4.38\left(\mathrm{~s}, 2 \mathrm{H}, \mathrm{S}-\mathrm{CH}_{2}\right), 7.25-7.64(\mathrm{~m}, 6 \mathrm{H}, \mathrm{Ar}-\mathrm{H}), 7.67-7.85(\mathrm{~m}, 1 \mathrm{H}, \mathrm{Ar}-\mathrm{H}), 8.09-8.29(\mathrm{~m}, 2 \mathrm{H}$, Ar-H), 8.64-8.69 (m, 1H, Ar-H), 10.40 (s, 1H, N-H). ${ }^{13} \mathrm{C}$ NMR (125 MHz, DMSO-d 6 ): $33.46\left(\mathrm{CH}_{2}, \mathrm{~S}^{\left.-\mathrm{CH}_{2}\right),}, 36.37\right.$ $\left(\mathrm{CH}_{2}, \mathrm{~S}-\mathrm{CH}_{2} \mathrm{CO}\right), 112.86(\mathrm{CH}, \mathrm{C}-5$, pyrimidine), $113.18(\mathrm{CH}, \mathrm{C}-6,3$-chlorophenyl), $127.69(\mathrm{CH}, \mathrm{C}-2,3-$ chlorophenyl), 128.80 ( $\mathrm{CH}, \mathrm{C}-4,4$-chlorophenyl), 129.17 (CH, C-2, 4-chlorophenyl), 129.33 (CH, C-6, 4chlorophenyl), 129.48 ( $\mathrm{CH}, \mathrm{C}-3$, 4-chlorophenyl), 129.56 (CH, C-5, 4-chlorophenyl), 131.05 (CH, C-5, 3chlorophenyl), 133.47 (C, C-1, 4-chlorophenyl), 134.80 (C, C-4, 4-chlorophenyl), 136.63 (C, C-3, 3-chlorophenyl), 136.81 (C, C-1, 3-chlorophenyl), 158.90 ( $\mathrm{CH}, \mathrm{C}-6$, pyrimidine), 159.22 (C, C-2, oxadiazole), 162.53 (C, C-5, oxadiazole), 164.47 (C, C-4, pyrimidine), 171.07 (C, $\mathrm{C}=\mathrm{O}), 171.46$ (C, C-2, pyrimidine). HRMS (ESI) (m/z) $[\mathrm{M}+\mathrm{H}]^{+}$calcd. for $\mathrm{C}_{21} \mathrm{H}_{15} \mathrm{Cl}_{2} \mathrm{~N}_{5} \mathrm{O}_{2} \mathrm{~S}_{2}$ : 504.0117, found: 504.0093.

\section{2-[(5-((4-(4-Chlorophenyl)pyrimidin-2-yl)thio)methyl)-1,3,4-oxadiazol-2-yl)thio]-N-(2,6-dimethylphenyl)acetamide} (4)

M.p. $257-258{ }^{\circ} \mathrm{C}$. Yield $63 \%$. IR $v_{\max }\left(\mathrm{cm}^{-1}\right): 3232.70$ (N-H stretching), 3072.60 (Aromatic C-H stretching), 2976.16, 2922.16, 2858.51 (Aliphatic C-H stretching), 1666.50 ( $\mathrm{C}=\mathrm{O}$ stretching), 1597.06, 1556.55, 1537.27, 1489.05 (N-H bending, $\mathrm{C}=\mathrm{N}$ and $\mathrm{C}=\mathrm{C}$ stretching), 1425.40, 1396.46, 1342.46, 1259.52, 1197.79, 1089.78, 1012.63 (C-H bending, C-N, C-O stretching and aromatic C-H in plane bending), 819.75, 773.46, 731.02, 667.37 (Aromatic C-H out of plane bending and C-S stretching). ${ }^{1} \mathrm{H}$ NMR $\left(500 \mathrm{MHz}\right.$, DMSO-d $\left.d_{6}\right): 1.98\left(\mathrm{~s}, 3 \mathrm{H}, \mathrm{CH}_{3}\right), 2.07$ $\left(\mathrm{s}, 3 \mathrm{H}, \mathrm{CH}_{3}\right), 4.16\left(\mathrm{~s}, 2 \mathrm{H}, \mathrm{S}-\mathrm{CH}_{2} \mathrm{CO}\right), 4.39\left(\mathrm{~s}, 2 \mathrm{H}, \mathrm{S}-\mathrm{CH}_{2}\right), 7.01-7.24(\mathrm{~m}, 3 \mathrm{H}, \mathrm{Ar}-\mathrm{H}), 7.50(\mathrm{~d}, J=7.30 \mathrm{~Hz}, 1 \mathrm{H}, \mathrm{Ar}-\mathrm{H})$, $7.56(\mathrm{~d}, J=7.75 \mathrm{~Hz}, 1 \mathrm{H}, \mathrm{Ar}-\mathrm{H}), 7.76(\mathrm{~s}, 1 \mathrm{H}, \mathrm{Ar}-\mathrm{H}), 8.13-8.27(\mathrm{~m}, 2 \mathrm{H}, \mathrm{Ar}-\mathrm{H}), 8.67$ (s, $1 \mathrm{H}, \mathrm{Ar}-\mathrm{H}), 10.41(\mathrm{~s}, 1 \mathrm{H}, \mathrm{N}-$ H). ${ }^{13} \mathrm{C}$ NMR (125 MHz, DMSO-d 6 ): $17.65\left(\mathrm{CH}_{3}\right), 18.52\left(\mathrm{CH}_{3}\right), 33.12\left(\mathrm{CH}_{2}, \mathrm{~S}-\mathrm{CH}_{2}\right), 35.67\left(\mathrm{CH}_{2}, \mathrm{~S}-\mathrm{CH}_{2} \mathrm{CO}\right), 113.14$ (CH, C-5, pyrimidine), $126.97(\mathrm{CH}, \mathrm{C}-4,2$,6-dimethylphenyl), $128.07(\mathrm{CH}, \mathrm{C}-3,2,6$-dimethylphenyl), 128.14 (CH, C-5, 2,6-dimethylphenyl), 128.63 ( $\mathrm{CH}, \mathrm{C}-2,4$-chlorophenyl), $128.69(\mathrm{CH}, \mathrm{C}-6,4$-chlorophenyl), 129.43 (CH, C-3, 4-chlorophenyl), 129.50 ( $\mathrm{CH}, \mathrm{C}-5$, 4-chlorophenyl), 129.54 (C, C-2, 2,6-dimethylphenyl), 129.65 (C, C-6, 2,6-dimethylphenyl), 134.83 (C, C-1, 4-chlorophenyl), 135.63 (C, C-4, 4-chlorophenyl), 136.28 (C, C-1, 2,6dimethylphenyl), 157.30 ( $\mathrm{CH}, \mathrm{C}-6$, pyrimidine), 159.18 (C, C-2, oxadiazole), 162.50 (C, C-5, oxadiazole), 164.43 (C, C-4, pyrimidine), $169.78(\mathrm{C}, \mathrm{C}=\mathrm{O}), 171.40$ (C, C-2, pyrimidine). HRMS (ESI) (m/z) $[\mathrm{M}+\mathrm{H}]^{+}$calcd. for $\mathrm{C}_{23} \mathrm{H}_{20} \mathrm{ClN}_{5} \mathrm{O}_{2} \mathrm{~S}_{2}: 498.0820$, found: 498.0809 .

\section{2-[(5-((4-(4-Chlorophenyl)pyrimidin-2-yl)thio)methyl)-1,3,4-oxadiazol-2-yl)thio]-N-(1,3-benzodioxol-5-} ylmethyl)acetamide (5)

M.p. $269-270{ }^{\circ} \mathrm{C}$. Yield 56\%. IR $\mathrm{v}_{\max }\left(\mathrm{cm}^{-1}\right): 3292.49$ (N-H stretching), 3062.96 (Aromatic C-H stretching), 2927.94 (Aliphatic C-H stretching), 1660.71 ( $\mathrm{C}=\mathrm{O}$ stretching), 1629.85, 1558.48, 1533.41, 1487.12 (N-H bending, $\mathrm{C}=\mathrm{N}$ and $\mathrm{C}=\mathrm{C}$ stretching), 1427.32, 1388.75, 1363.67, 1342.46, 1327.03, 1247.94, 1197.79, 1168.86, 1089.78, 1033.85, 1010.70, 925.83, 875.68, 819.75, 773.46, 729.09, 686.66 (Aromatic C-H out of plane bending and C-S stretching). ${ }^{1} \mathrm{H}$ NMR $\left(500 \mathrm{MHz}, \mathrm{DMSO}-d_{6}\right): 4.08\left(\mathrm{~s}, 2 \mathrm{H}, \mathrm{S}-\mathrm{CH}_{2} \mathrm{CO}\right), 4.38\left(\mathrm{~s}, 2 \mathrm{H}, \mathrm{S}-\mathrm{CH}_{2}\right), 4.73\left(\mathrm{~s}, 2 \mathrm{H}, \mathrm{NH}-\mathrm{CH}_{2}\right)$, $5.96\left(\mathrm{~s}, 2 \mathrm{H}, \mathrm{O}-\mathrm{CH}_{2}-\mathrm{O}\right), 6.79(\mathrm{~d}, J=8.05 \mathrm{~Hz}, 2 \mathrm{H}, \mathrm{Ar}-\mathrm{H}), 6.85(\mathrm{~d}, J=8.50 \mathrm{~Hz}, 1 \mathrm{H}, \mathrm{Ar}-\mathrm{H}), 7.54(\mathrm{~d}, J=8.55 \mathrm{~Hz}, 2 \mathrm{H}, \mathrm{Ar}-$ $\mathrm{H}), 7.60(\mathrm{~d}, J=8.50 \mathrm{~Hz}, 1 \mathrm{H}, \mathrm{Ar}-\mathrm{H}), 8.20(\mathrm{~d}, J=8.55 \mathrm{~Hz}, 2 \mathrm{H}, \mathrm{Ar}-\mathrm{H}), 8.66-8.72(\mathrm{~m}, 1 \mathrm{H}, \mathrm{Ar}-\mathrm{H}), 10.40(\mathrm{~s}, 1 \mathrm{H}, \mathrm{N}-\mathrm{H})$. ${ }^{13} \mathrm{C}$ NMR (125 MHz, DMSO-d 6 ): $33.29\left(\mathrm{CH}_{2}, \mathrm{~S}-\mathrm{CH}_{2}\right), 36.10\left(\mathrm{CH}_{2}, \mathrm{~S}-\mathrm{CH}_{2} \mathrm{CO}\right), 42.89\left(\mathrm{CH}_{2}, \mathrm{NH}-\mathrm{CH}_{2}\right), 101.40\left(\mathrm{CH}_{2}\right.$,

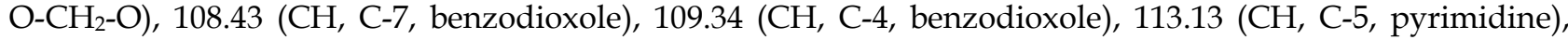
122.25 (CH, C-6, benzodioxole), 129.37 (CH, C-2, 4-chlorophenyl), $129.46(\mathrm{CH}, \mathrm{C}-6$, 4-chlorophenyl), 129.50 
(CH, C-3, 4-chlorophenyl), 129.55 (CH, C-5, 4-chlorophenyl), 130.07 (C, C-1, benzodioxole), 133.12 (C, C-1, 4chlorophenyl), 134.81 (C, C-4, 4-chlorophenyl), 147.13 (C, C-3a, benzodioxole), 147.61 (C, C-7a, benzodioxole), 156.70 (CH, C-6, pyrimidine), 159.21 (C, C-2, oxadiazole), 162.49 (C, C-5, oxadiazole), 164.63 (C, C-4, pyrimidine), $169.42(\mathrm{C}, \mathrm{C}=\mathrm{O}), 171.70$ (C, C-2, pyrimidine). HRMS (ESI) $(\mathrm{m} / \mathrm{z})[\mathrm{M}+\mathrm{H}]^{+}$calcd. for $\mathrm{C}_{23} \mathrm{H}_{18} \mathrm{ClN}_{5} \mathrm{O}_{4} \mathrm{~S}_{2}:$ 528.0561, found: 528.0543 .

\section{2-[(5-(((4-(4-Chlorophenyl)pyrimidin-2-yl)thio)methyl)-1,3,4-oxadiazol-2-yl)thio]-1-morpholinoethan-1-one (6)}

M.p. 292-293 ${ }^{\circ} \mathrm{C}$. Yield 65\%. IR $\mathrm{v}_{\max }\left(\mathrm{cm}^{-1}\right)$ : 3232.70 (N-H stretching), 3089.96, 3061.03, 3010.88 (Aromatic C-H stretching), 2974.23, 2926.01, 2860.43 (Aliphatic C-H stretching), 1641.42 (C=O stretching), 1624.06, 1597.06, 1556.55, 1535.34, 1463.97 ( $\mathrm{N}-\mathrm{H}$ bending, $\mathrm{C}=\mathrm{N}$ and $\mathrm{C}=\mathrm{C}$ stretching), 1427.32, 1398.39, 1348.24, 1300.02, $1276.88,1220.94,1199.72,1180.44,1151.50,1112.93,1097.50,1068.56,1039.63,1012.63$ (C-H bending, C-N, C-O stretching and aromatic C-H in plane bending), 974.05, 956.69, 902.69, 852.54, 819.75, 773.46, 756.10, 731.02, 684.73, 669.30 (Aromatic C-H out of plane bending and C-S stretching). ${ }^{1} \mathrm{H}$ NMR (500 MHz, DMSO- $\left.d_{6}\right): 3.44(\mathrm{t}$, $\left.J=5.25,4.35,9.60 \mathrm{~Hz}, 4 \mathrm{H}, \mathrm{H}_{2} \mathrm{C}-\mathrm{N}-\mathrm{CH}_{2}\right), 3.54\left(\mathrm{t}, J=4.45,9.40 \mathrm{~Hz}, 2 \mathrm{H}, \mathrm{H}_{2} \mathrm{C}-\mathrm{O}-\mathrm{CH}_{2}\right), 3.58(\mathrm{t}, J=4.40,4.70,9.10 \mathrm{~Hz}$, $\left.2 \mathrm{H}, \mathrm{H}_{2} \mathrm{C}-\mathrm{O}-\mathrm{CH}_{2}\right), 4.42\left(\mathrm{~s}, 2 \mathrm{H}, \mathrm{S}-\mathrm{CH}_{2} \mathrm{CO}\right), 4.75\left(\mathrm{~s}, 2 \mathrm{H}, \mathrm{S}-\mathrm{CH}_{2}\right), 7.60(\mathrm{~d}, J=8.65 \mathrm{~Hz}, 2 \mathrm{H}, \mathrm{Ar}-\mathrm{H}), 7.84(\mathrm{~d}, J=5.30 \mathrm{~Hz}$, $1 \mathrm{H}, \mathrm{Ar}-\mathrm{H}), 8.23(\mathrm{~d}, J=8.65 \mathrm{~Hz}, 2 \mathrm{H}, \mathrm{Ar}-\mathrm{H}), 8.72(\mathrm{~d}, J=5.30 \mathrm{~Hz}, 1 \mathrm{H}, \mathrm{Ar}-\mathrm{H}) .{ }^{13} \mathrm{C}$ NMR $\left(125 \mathrm{MHz}, \mathrm{DMSO}-d_{6}\right): 24.59$ $\left(\mathrm{CH}_{2}, \mathrm{~S}-\mathrm{CH}_{2}\right), 36.85\left(\mathrm{CH}_{2}, \mathrm{~S}-\mathrm{CH}_{2} \mathrm{CO}\right), 42.50\left(\mathrm{CH}_{2}, \underline{\mathrm{H}_{2}} \mathrm{C}-\mathrm{N}-\mathrm{CH}_{2}\right), 46.19\left(\mathrm{CH}_{2}, \mathrm{H}_{2} \mathrm{C}-\mathrm{N}-\mathrm{CH}_{2}\right), 66.31\left(2 \mathrm{CH}_{2}, \mathrm{H}_{2} \mathrm{C}-\mathrm{O}-\right.$ $\left.\mathrm{CH}_{2}\right), 113.60$ ( $\mathrm{CH}, \mathrm{C}-5$, pyrimidine), 129.47 (2CH, C-2 and C-6, 4-chlorophenyl), 129.55 (2CH, C-3 and C-5, 4chlorophenyl), 134.59 (C, C-1, 4-chlorophenyl), 137.00 (C, C-4, 4-chlorophenyl), 159.54 (CH, C-6, pyrimidine), 162.63 (C, C-2, oxadiazole), 164.12 (C, C-5, oxadiazole), 165.15 (C, C-4, pyrimidine), 165.66 (C, C=O), 169.43 (C, $\mathrm{C}-2$, pyrimidine). HRMS (ESI) $(\mathrm{m} / \mathrm{z})[\mathrm{M}+\mathrm{H}]^{+}$calcd. for $\mathrm{C}_{19} \mathrm{H}_{18} \mathrm{ClN}_{5} \mathrm{O}_{3} \mathrm{~S}_{2}: 464.0612$, found: 464.0589 .

\section{2-[(5-(((4-(4-Chlorophenyl)pyrimidin-2-yl)thio)methyl)-1,3,4-oxadiazol-2-yl)thio]-N,N-diethylacetamide (7)}

M.p. 203-204 ${ }^{\circ} \mathrm{C}$. Yield 74\%. IR $\mathrm{v}_{\max }\left(\mathrm{cm}^{-1}\right)$ : 3194.12 (N-H stretching), 3091.89 (Aromatic C-H stretching), 2978.09, 2929.87, 2910.58, 2875.86 (Aliphatic C-H stretching), 1653.00 (C=O stretching), 1629.85, 1564.27, 1537.27, 1479.40 (N-H bending, $\mathrm{C}=\mathrm{N}$ and $\mathrm{C}=\mathrm{C}$ stretching), 1435.04, 1394.53, 1344.38, 1336.67, 1215.15, 1138.00, 1087.85, 1066.64, 1004.91 (C-H bending, C-N, C-O stretching and aromatic C-H in plane bending), 975.98, 952.84, 920.05, 893.04, 821.68, 773.46, 731.02, 665.44 (Aromatic C-H out of plane bending and C-S stretching). ${ }^{1} \mathrm{H}$ NMR $\left(500 \mathrm{MHz}\right.$, DMSO- $\left.d_{6}\right): 1.00\left(\mathrm{t}, J=7.05,7.10,14.15 \mathrm{~Hz}, 3 \mathrm{H}, \mathrm{CH}_{3}\right), 1.11\left(\mathrm{t}, J=7.10,14.20 \mathrm{~Hz}, 3 \mathrm{H}, \mathrm{CH}_{3}\right)$, $3.26\left(\mathrm{q}, J=7.15,14.25 \mathrm{~Hz}, 2 \mathrm{H}, \mathrm{CH}_{2}\right), 3.31\left(\mathrm{q}, J=7.10,14.25 \mathrm{~Hz}, 2 \mathrm{H}, \mathrm{CH}_{2}\right), 4.39$ (s, 2H, S-CH $\mathrm{CH}_{2} \mathrm{CO}, 4.75$ (s, 2H, S$\left.\mathrm{CH}_{2}\right), 7.60(\mathrm{~d}, J=8.60 \mathrm{~Hz}, 2 \mathrm{H}, \mathrm{Ar}-\mathrm{H}), 7.85(\mathrm{~d}, J=5.30 \mathrm{~Hz}, 1 \mathrm{H}, \mathrm{Ar}-\mathrm{H}), 8.23(\mathrm{~d}, J=8.65 \mathrm{~Hz}, 2 \mathrm{H}, \mathrm{Ar}-\mathrm{H}), 8.72(\mathrm{~d}, J=$ $5.25 \mathrm{~Hz}, 1 \mathrm{H}, \mathrm{Ar}-\mathrm{H}) .{ }^{13} \mathrm{C}$ NMR $\left(125 \mathrm{MHz}, \mathrm{DMSO}-d_{6}\right): 13.29\left(\mathrm{CH}_{3}\right), 14.32\left(\mathrm{CH}_{3}\right), 24.57\left(\mathrm{CH}_{2}, \mathrm{~S}_{-} \mathrm{CH}_{2}\right), 37.14\left(\mathrm{CH}_{2}\right.$, $\left.\mathrm{S}-\mathrm{CH}_{2} \mathrm{CO}\right), 42.31\left(2 \mathrm{CH}_{2}\right), 113.61(\mathrm{CH}, \mathrm{C}-5$, pyrimidine $), 129.48(2 \mathrm{CH}, \mathrm{C}-2$ and $\mathrm{C}-6,4$-chlorophenyl), 129.56 (2CH, C-3 and C-5, 4-chlorophenyl), 134.60 (C, C-1, 4-chlorophenyl), 137.00 (C, C-4, 4-chlorophenyl), 159.55 (CH, C-6, pyrimidine), 162.64 (C, C-2, oxadiazole), 164.36 (C, C-5, oxadiazole), 165.24 (C, C-4, pyrimidine), $165.54(\mathrm{C}, \mathrm{C}=\mathrm{O}), 169.44\left(\mathrm{C}, \mathrm{C}-2\right.$, pyrimidine). HRMS (ESI) (m/z) $[\mathrm{M}+\mathrm{H}]^{+}$calcd. for $\mathrm{C}_{19} \mathrm{H}_{20} \mathrm{ClN}_{5} \mathrm{O}_{2} \mathrm{~S}_{2}: 450.0820$, found: 450.0808 .

\section{2-[(5-(((4-(4-Chlorophenyl)pyrimidin-2-yl)thio)methyl)-1,3,4-oxadiazol-2-yl)thio]-N-(benzothiazol-2-yl)acetamide (8)}

M.p. $274-275^{\circ} \mathrm{C}$. Yield $67 \%$. IR $v_{\max }\left(\mathrm{cm}^{-1}\right): 3174.83$ (N-H stretching), 3061.03 (Aromatic C-H stretching), 2962.66, 2916.37, 2848.86 (Aliphatic C-H stretching), 1678.07 (C=O stretching), 1602.85, 1564.27, 1533.41, $1487.12(\mathrm{~N}-\mathrm{H}$ bending, $\mathrm{C}=\mathrm{N}$ and $\mathrm{C}=\mathrm{C}$ stretching), 1435.04, 1382.96, 1336.67, 1261.45, 1205.51, 1174.65, 1089.78, 1012.63 (C-H bending, C-N, C-O stretching and aromatic C-H in plane bending), 871.82, 821.68, 759.95, 729.09, 667.37 (Aromatic C-H out of plane bending and C-S stretching). ${ }^{1} \mathrm{H}$ NMR $\left(500 \mathrm{MHz}, \mathrm{DMSO}-d_{6}\right): 4.40$ (s, $2 \mathrm{H}$, S$\left.\mathrm{CH}_{2} \mathrm{CO}\right), 4.75\left(\mathrm{~s}, 2 \mathrm{H}, \mathrm{S}-\mathrm{CH}_{2}\right), 7.31(\mathrm{t}, J=7.60,7.45,15.05 \mathrm{~Hz}, 1 \mathrm{H}, \mathrm{Ar}-\mathrm{H}), 7.44(\mathrm{t}, J=7.45,7.55,15.00 \mathrm{~Hz}, 1 \mathrm{H}, \mathrm{Ar}-$ $\mathrm{H}), 7.57(\mathrm{~d}, J=8.45 \mathrm{~Hz}, 2 \mathrm{H}, \mathrm{Ar}-\mathrm{H}), 7.75(\mathrm{~d}, J=7.95 \mathrm{~Hz}, 1 \mathrm{H}, \mathrm{Ar}-\mathrm{H}), 7.77-7.79(\mathrm{~m}, 1 \mathrm{H}, \mathrm{Ar}-\mathrm{H}), 7.95(\mathrm{~d}, J=7.90 \mathrm{~Hz}$, 1H, Ar-H), $8.16(\mathrm{~d}, J=8.50 \mathrm{~Hz}, 2 \mathrm{H}, \mathrm{Ar}-\mathrm{H}), 8.68(\mathrm{~d}, J=5.25 \mathrm{~Hz}, 1 \mathrm{H}, \mathrm{Ar}-\mathrm{H}), 10.39$ (s, 1H, N-H). ${ }^{13} \mathrm{C}$ NMR (125 MHz, DMSO- $\left.d_{6}\right)$ : $24.62\left(\mathrm{CH}_{2}, \mathrm{~S}-\mathrm{CH}_{2}\right), 36.06\left(\mathrm{CH}_{2}, \mathrm{~S}_{-} \mathrm{CH}_{2} \mathrm{CO}\right), 113.61(\mathrm{CH}, \mathrm{C}-5$, pyrimidine $), 121.13(\mathrm{CH}, \mathrm{C}-4$, benzothiazole), $122.19(\mathrm{CH}, \mathrm{C}-7$, benzothiazole), $124.18(\mathrm{CH}, \mathrm{C}-6$, benzothiazole), $126.65(\mathrm{CH}, \mathrm{C}-5$, benzothiazole), 129.32 ( $\mathrm{CH}, \mathrm{C}-2$, 4-chlorophenyl), 129.41 (CH, C-6, 4-chlorophenyl), 129.52 (2CH, C-3 and C-5, 4-chlorophenyl), 131.91 (C, C-7a, benzothiazole), 134.56 (C, C-1, 4-chlorophenyl), 139.96 (C, C-4, 4chlorophenyl), 148.86 (C, C-3a, benzothiazole), 158.09 (CH, C-6, pyrimidine), 159.47 (C, C-2, oxadiazole), 162.66 (C, C-5, oxadiazole), 163.61 (C, C-4, pyrimidine), 166.06 (C, C=O), 166.68 (C, C-2, pyrimidine), 169.39 (C, C-2, benzothiazole). HRMS (ESI) (m/z) [M+H] calcd. for $\mathrm{C}_{22} \mathrm{H}_{15} \mathrm{ClN}_{6} \mathrm{O}_{2} \mathrm{~S}_{3}: 527.0180$, found: 527.0148. 
2-[(5-((4-(4-Chlorophenyl)pyrimidin-2-yl)thio)methyl)-1,3,4-oxadiazol-2-yl)thio]-N-(6-fluorobenzothiazol-2yl)acetamide (9)

M.p. $284-285^{\circ} \mathrm{C}$. Yield 54\%. IR $v_{\max }\left(\mathrm{cm}^{-1}\right)$ : 3248.13 (N-H stretching), 3149.76, 3074.53 (Aromatic C-H stretching), 2989.66, 2962.66, 2918.30, 2872.01 (Aliphatic C-H stretching), 1678.07 (C=O stretching), 1610.56, $1562.34,1533.41,1489.05,1456.26(\mathrm{~N}-\mathrm{H}$ bending, $\mathrm{C}=\mathrm{N}$ and $\mathrm{C}=\mathrm{C}$ stretching), 1435.04, 1382.96, 1338.60, 1259.52, $1247.94,1203.58,1174.65,1159.22,1091.71,1012.63$ (C-H bending, C-N, C-O stretching and aromatic C-H in plane bending), 995.27, 914.26, 825.53, 815.59, 773.46, 748.38, 727.16, 704.02, 682.80, 665.44 (Aromatic C-H out of plane bending and C-S stretching). ${ }^{1} \mathrm{H}$ NMR (500 MHz, DMSO- $\left.d_{6}\right): 4.38$ (s, 2H, S- $\left.\mathrm{CH}_{2} \mathrm{CO}\right), 4.74(\mathrm{~s}, 2 \mathrm{H}, \mathrm{S}-$ $\left.\mathrm{CH}_{2}\right), 7.27(\mathrm{td}, J=2.65,2.60,9.03,18.05 \mathrm{~Hz}, 1 \mathrm{H}, \mathrm{Ar}-\mathrm{H}), 7.57(\mathrm{~d}, J=8.55 \mathrm{~Hz}, 2 \mathrm{H}, \mathrm{Ar}-\mathrm{H}), 7.74(\mathrm{~d}, J=8.85 \mathrm{~Hz}, 1 \mathrm{H}$, Ar-H), $7.79(\mathrm{~d}, J=5.30 \mathrm{~Hz}, 1 \mathrm{H}, \mathrm{Ar}-\mathrm{H}), 7.84(\mathrm{~d}, J=8.63 \mathrm{~Hz}, 1 \mathrm{H}, \mathrm{Ar}-\mathrm{H}), 8.16(\mathrm{~d}, J=8.60 \mathrm{~Hz}, 2 \mathrm{H}, \mathrm{Ar}-\mathrm{H}), 8.68(\mathrm{~d}, J=$ $5.25 \mathrm{~Hz}, 1 \mathrm{H}, \mathrm{Ar}-\mathrm{H}), 10.40$ (s, 1H, N-H). ${ }^{13} \mathrm{C}$ NMR (125 MHz, DMSO-d $)$ : $24.60\left(\mathrm{CH}_{2}, \mathrm{~S}_{-} \mathrm{CH}_{2}\right), 36.08\left(\mathrm{CH}_{2}\right.$, S$\mathrm{CH}_{2} \mathrm{CO}$ ), 108.61 (d, J= 26.68 Hz, CH, C-7, benzothiazole), $113.60(\mathrm{CH}, \mathrm{C}-5$, pyrimidine), 114.72 (d, J= 24.33 Hz, $\mathrm{CH}, \mathrm{C}-5$, benzothiazole), $122.21(\mathrm{~d}, \mathrm{~J}=9.09 \mathrm{~Hz}, \mathrm{CH}, \mathrm{C}-4$, benzothiazole), $129.40(2 \mathrm{CH}, \mathrm{C}-2$ and C-6, 4chlorophenyl), 129.50 (2CH, C-3 and C-5, 4-chlorophenyl), 133.19 (d, J=11.09 Hz, C, C-7a, benzothiazole), 134.55 (C, C-1, 4-chlorophenyl), 136.95 (C, C-4, 4-chlorophenyl), 145.66 (C, C-3a, benzothiazole), 158.25 (d, J= $12.65 \mathrm{~Hz}, \mathrm{C}, \mathrm{C}-6$, pyrimidine), 159.46 (CH, C-6, benzothiazole), 160.11 (C, C-2, oxadiazole), 162.65 (C, C-5, oxadiazole), 163.63 (C, C-4, pyrimidine), 166.05 (C, C=O), 166.83 (C, C-2, pyrimidine), 169.38 (C, C-2, benzothiazole). HRMS (ESI) (m/z) [M+H] ${ }^{+}$calcd. for $\mathrm{C}_{22} \mathrm{H}_{14} \mathrm{ClFN}_{6} \mathrm{O}_{2} \mathrm{~S}_{3}: 545.0086$, found: 545.0080.

2-[(5-((4-(4-Chlorophenyl)pyrimidin-2-yl)thio)methyl)-1,3,4-oxadiazol-2-yl)thio]-N-(6-chlorobenzothiazol-2yl)acetamide (10)

M.p. 220-221 ${ }^{\circ} \mathrm{C}$. Yield 51\%. IR $\mathrm{v}_{\max }\left(\mathrm{cm}^{-1}\right): 3246.20,3172.90(\mathrm{~N}-\mathrm{H}$ stretching), 3138.18, 3057.17 (Aromatic C-H stretching), 2987.74, 2962.66, 2920.23, 2856.58 (Aliphatic C-H stretching), 1680.00 (C=O stretching), $1600.92,1562.34,1531.48,1487.12$ ( $\mathrm{N}-\mathrm{H}$ bending, $\mathrm{C}=\mathrm{N}$ and $\mathrm{C}=\mathrm{C}$ stretching), 1435.04, 1396.46, 1381.03, 1338.60, 1261.45, 1234.44, 1203.58, 1174.65, 1155.36, 1091.71, 1012.63 (C-H bending, C-N, C-O stretching and aromatic C-H in plane bending), 995.27, 823.60, 808.17, 763.81, 748.38, 727.16, 688.59, 665.44 (Aromatic C-H out of plane bending and C-S stretching). ${ }^{1} \mathrm{H}$ NMR $\left(500 \mathrm{MHz}\right.$, DMSO-d $\left.d_{6}\right)$ : 4.38 (s, $\left.2 \mathrm{H}, \mathrm{S}-\mathrm{CH}_{2} \mathrm{CO}\right), 4.74\left(\mathrm{~s}, 2 \mathrm{H}, \mathrm{S}-\mathrm{CH}_{2}\right), 7.44-$ $7.79(\mathrm{~m}, 5 \mathrm{H}, \mathrm{Ar}-\mathrm{H}), 8.06-8.16(\mathrm{~m}, 3 \mathrm{H}, \mathrm{Ar}-\mathrm{H}), 8.68(\mathrm{~s}, 1 \mathrm{H}, \mathrm{Ar}-\mathrm{H}), 10.40(\mathrm{~s}, 1 \mathrm{H}, \mathrm{N}-\mathrm{H}) .{ }^{13} \mathrm{C}$ NMR $(125 \mathrm{MHz}, \mathrm{DMSO}-$ $\left.d_{6}\right): 24.59\left(\mathrm{CH}_{2}, \mathrm{~S}-\mathrm{CH}_{2}\right), 36.27\left(\mathrm{CH}_{2}, \mathrm{~S}-\mathrm{CH}_{2} \mathrm{CO}\right), 113.52(\mathrm{CH}, \mathrm{C}-5$, pyrimidine), $115.30(\mathrm{CH}, \mathrm{C}-4$, benzothiazole $)$, 121.57 (CH, C-7, benzothiazole), 125.82 ( $\mathrm{CH}, \mathrm{C}-5$, benzothiazole), 128. 52 (C, C-6, benzothiazole), $129.32(2 \mathrm{CH}$, C-2 and C-6, 4-chlorophenyl), 129.46 (2CH, C-3 and C-5, 4-chlorophenyl), 133.20 (C, C-7a, benzothiazole), 134.46 (C, C-1, 4-chlorophenyl), 137.45 (C, C-4, 4-chlorophenyl), 148.16 (C, C-3a, benzothiazole), 159.52 (CH, C-6, pyrimidine), 159.67 (C, C-2, oxadiazole), 160.64 (C, C-5, oxadiazole), 162.56 (C, C-4, pyrimidine), 166.10 (C, C=O), 167.32 (C, C-2, pyrimidine), 169.42 (C, C-2, benzothiazole). HRMS (ESI) (m/z) [M+H] $]^{+}$calcd. for $\mathrm{C}_{22} \mathrm{H}_{14} \mathrm{Cl}_{2} \mathrm{~N}_{6} \mathrm{O}_{2} \mathrm{~S}_{3}: 560.9790$, found: 560.9763 .

2-[(5-(((4-(4-Chlorophenyl)pyrimidin-2-yl)thio)methyl)-1,3,4-oxadiazol-2-yl)thio]-N-(6-bromobenzothiazol-2yl)acetamide (11)

M.p. 177-179 ${ }^{\circ} \mathrm{C}$. Yield 59\%. IR $\mathrm{v}_{\max }\left(\mathrm{cm}^{-1}\right)$ : 3174.83 (N-H stretching), 3078.39, 3053.32 (Aromatic C-H stretching), 2976.16, 2926.01 (Aliphatic C-H stretching), 1681.93 (C=O stretching), 1595.13, 1562.34, 1531.48, 1475.54 (N-H bending, $\mathrm{C}=\mathrm{N}$ and $\mathrm{C}=\mathrm{C}$ stretching), 1425.40, 1396.46, 1334.74, 1265.30, 1203.58, 1170.79, 1089.78, 1012.63 (C-H bending, C-N, C-O stretching and aromatic C-H in plane bending), 991.41, 812.03, 771.53, 746.45, 731.02, 684.73, 665.44 (Aromatic C-H out of plane bending and C-S stretching). ${ }^{1} \mathrm{H}$ NMR (500 MHz, DMSO- $d_{6}$ ): $4.38\left(\mathrm{~s}, 2 \mathrm{H}, \mathrm{S}-\mathrm{CH}_{2} \mathrm{CO}\right), 4.74\left(\mathrm{~s}, 2 \mathrm{H}, \mathrm{S}-\mathrm{CH}_{2}\right), 7.55(\mathrm{~d}, J=8.50 \mathrm{~Hz}, 2 \mathrm{H}, \mathrm{Ar}-\mathrm{H}), 7.64(\mathrm{~d}, J=8.55 \mathrm{~Hz}, 2 \mathrm{H}, \mathrm{Ar}-\mathrm{H}), 7.76$ $(\mathrm{d}, J=5.30 \mathrm{~Hz}, 1 \mathrm{H}, \mathrm{Ar}-\mathrm{H}), 8.13-8.16(\mathrm{~m}, 3 \mathrm{H}, \mathrm{Ar}-\mathrm{H}), 8.67(\mathrm{~d}, J=5.20 \mathrm{~Hz}, 1 \mathrm{H}, \mathrm{Ar}-\mathrm{H}), 10.39(\mathrm{~s}, 1 \mathrm{H}, \mathrm{N}-\mathrm{H}) .{ }^{13} \mathrm{C}$ NMR (125 MHz, DMSO- $\left.d_{6}\right): 24.61\left(\mathrm{CH}_{2}, \mathrm{~S}-\mathrm{CH}_{2}\right), 36.30\left(\mathrm{CH}_{2}, \mathrm{~S}-\mathrm{CH}_{2} \mathrm{CO}\right), 113.57(\mathrm{CH}, \mathrm{C}-5$, pyrimidine), $115.93(\mathrm{CH}, \mathrm{C}-$ 4, benzothiazole), 122.58 (C, C-6, benzothiazole), 124.62 ( $\mathrm{CH}, \mathrm{C}-7$, benzothiazole), 129.29 ( $\mathrm{CH}, \mathrm{C}-5$, benzothiazole), 129.36 (CH, C-2, 4-chlorophenyl), 129.43 (CH, C-6, 4-chlorophenyl), 129.48 (CH, C-3, 4chlorophenyl), 129.52 (CH, C-5, 4-chlorophenyl), 134.18 (C, C-7a, benzothiazole), 134.51 (C, C-1, 4chlorophenyl), 136.95 (C, C-4, 4-chlorophenyl), 148.19 (C, C-3a, benzothiazole), 159.42 (CH, C-6, pyrimidine), 159.53 (C, C-2, oxadiazole), 162.62 (C, C-5, oxadiazole), 163.69 (C, C-4, pyrimidine), 166.01 (C, C=O), 167.17 (C, C-2, pyrimidine), 169.37 (C, C-2, benzothiazole). HRMS (ESI) (m/z) $[\mathrm{M}+\mathrm{H}]^{+}$calcd. for $\mathrm{C}_{22} \mathrm{H}_{14} \mathrm{BrClN}_{6} \mathrm{O}_{2} \mathrm{~S}_{3}$ : 604.9285, found: 604.9262 . 
2-[(5-(((4-(4-Chlorophenyl)pyrimidin-2-yl)thio)methyl)-1,3,4-oxadiazol-2-yl)thio]-N-(6-methylbenzothiazol-2yl)acetamide (12)

M.p. 206-208 ${ }^{\circ} \mathrm{C}$. Yield 55\%. IR $\mathrm{v}_{\max }\left(\mathrm{cm}^{-1}\right)$ : 3250.05, 3182.55 (N-H stretching), 3072.60 (Aromatic C-H stretching), 2960.73, 2920.23, 2860.43 (Aliphatic C-H stretching), 1678.07 (C=O stretching), 1608.63, 1562.34, $1531.48,1487.12,1460.11(\mathrm{~N}-\mathrm{H}$ bending, $\mathrm{C}=\mathrm{N}$ and $\mathrm{C}=\mathrm{C}$ stretching), 1433.11, 1398.39, 1381.03, 1340.53, 1261.45, 1203.58, 1172.72, 1153.43, 1091.71, 1014.56 (C-H bending, C-N, C-O stretching and aromatic C-H in plane bending), 995.27, 896.90, 821.68, 773.46, 727.16, 665.44 (Aromatic C-H out of plane bending and C-S stretching). ${ }^{1} \mathrm{H}$ NMR (500 MHz, DMSO-d 6 ): 2.39 (s, 3H, CH $), 4.07\left(\mathrm{~s}, 2 \mathrm{H}, \mathrm{S}-\mathrm{CH}_{2} \mathrm{CO}\right), 4.74\left(\mathrm{~s}, 2 \mathrm{H}, \mathrm{S}-\mathrm{CH}_{2}\right), 7.24(\mathrm{~d}, J=7.30 \mathrm{~Hz}$, $1 \mathrm{H}, \mathrm{Ar}-\mathrm{H}), 7.57$ (d, J= $8.55 \mathrm{~Hz}, 2 \mathrm{H}, \mathrm{Ar}-\mathrm{H}), 7.62(\mathrm{~d}, J=8.25 \mathrm{~Hz}, 1 \mathrm{H}, \mathrm{Ar}-\mathrm{H}), 7.78$ (d, J= $5.30 \mathrm{~Hz}, 2 \mathrm{H}, \mathrm{Ar}-\mathrm{H}), 8.16(\mathrm{~d}$, $J=8.55 \mathrm{~Hz}, 2 \mathrm{H}, \mathrm{Ar}-\mathrm{H}), 8.68(\mathrm{~d}, J=5.25 \mathrm{~Hz}, 1 \mathrm{H}, \mathrm{Ar}-\mathrm{H}), 10.40(\mathrm{~s}, 1 \mathrm{H}, \mathrm{N}-\mathrm{H}) .{ }^{13} \mathrm{C}$ NMR $\left(125 \mathrm{MHz}, \mathrm{DMSO}-d_{6}\right): 21.44$ $\left(\mathrm{CH}_{3}\right), 24.60\left(\mathrm{CH}_{2}, \mathrm{~S}-\mathrm{CH}_{2}\right), 36.03\left(\mathrm{CH}_{2}, \mathrm{~S}-\mathrm{CH}_{2} \mathrm{CO}\right), 113.61(\mathrm{CH}, \mathrm{C}-5$, pyrimidine $), 120.76(\mathrm{CH}, \mathrm{C}-4$, benzothiazole), 121.76 ( $\mathrm{CH}, \mathrm{C}-7$, benzothiazole), 127.97 ( $\mathrm{CH}, \mathrm{C}-5$, benzothiazole), 129.41 (2CH, C-2 and C-6, 4chlorophenyl), 129.52 (2CH, C-3 and C-5, 4-chlorophenyl), 132.05 (C, C-7a, benzothiazole), 133.66 (C, C-1, 4chlorophenyl), 134.18 (C, C-1, 4-chlorophenyl), 134.56 (C, C-6, benzothiazole), 150.21 (C, C-3a, benzothiazole), 157.23 (CH, C-6, pyrimidine), 159.46 (C, C-2, oxadiazole), 162.65 (C, C-5, oxadiazole), 163.62 (C, C-4, pyrimidine), 166.05 (C, C=O), 166.50 (C, C-2, pyrimidine), 169.38 (C, C-2, benzothiazole). HRMS (ESI) (m/z) $[\mathrm{M}+\mathrm{H}]^{+}$calcd. for $\mathrm{C}_{23} \mathrm{H}_{17} \mathrm{ClN}_{6} \mathrm{O}_{2} \mathrm{~S}_{3}: 541.0336$, found: 541.0310.

\subsection{Biochemistry}

\subsubsection{Cell culture and drug treatment}

NIH/3T3 mouse embryonic fibroblast cells (ATCC ${ }^{\circledR}$ CRL-1658 ${ }^{\mathrm{TM}}$ ) were incubated in Dulbecco's Modified Eagle's Medium (DMEM) (Sigma, Deisenhofen, Germany) supplemented with $10 \%$ fetal calf serum (Gibco, Paisley, Scotland). A549 Human lung adenocarcinoma cells (ATCC ${ }^{\circledR}$ CCL-185 ${ }^{\mathrm{TM}}$ ) were incubated in $90 \%$ RPMI supplemented with 10\% fetal bovine serum (Gibco, Paisley, Scotland). All media were supplemented with $100 \mathrm{IU} / \mathrm{mL}$ penicillin-streptomycin (Gibco, Paisley, Scotland) and cells were incubated at $37^{\circ} \mathrm{C}$ in a humidified atmosphere of $95 \%$ air and $5 \% \mathrm{CO}_{2}$. Exponentially growing cells were plated at $2 \times 10^{4} \mathrm{cells} / \mathrm{mL}$ into 96-well microtiter tissue culture plates (Nunc, Denmark) and incubated for $24 \mathrm{~h}$ before the addition of the drugs (the optimum cell number for cytotoxicity assays was determined in preliminary experiments). The stock solutions of the compounds were prepared in dimethyl sulfoxide (DMSO; Sigma Aldrich, Poole, UK) and further dilutions were made with fresh culture medium (the concentration of DMSO in the final culture medium was $<0.1 \%$ which had no effect on the cell viability) [40].

\subsubsection{MTT assay}

The level of cellular 3-(4,5-dimethylthiazol-2-yl)-2,5-diphenyltetrazolium bromide (MTT) (SigmaAldrich, St. Louis, MO, USA) reduction was quantified as previously described in the literature [41] with small modifications [40]. Compounds 1-12 were investigated for their antiproliferative activity against A549 human lung adenocarcinoma cell line. NIH/3T3 Mouse embryonic fibroblast cells were used to evaluate the selectivity of the compounds.

After $24 \mathrm{~h}$ of preincubation, compounds 1-12 and cisplatin (positive control) were added to give final concentration in the range 3.9-500 $\mu \mathrm{g} / \mathrm{mL}$ and the cells were incubated for $24 \mathrm{~h}$. At the end of this period, MTT was added to a final concentration of $0.5 \mathrm{mg} / \mathrm{mL}$ and the cells were incubated for $4 \mathrm{~h}$ at $37^{\circ} \mathrm{C}$. After the medium was removed, the formazan crystals formed by MTT metabolism were solubilized by addition of 200 $\mu \mathrm{L}$ DMSO to each well and absorbance was read at $540 \mathrm{~nm}$ with a microtiter plate spectrophotometer (Bio-Tek plate reader, Winooski, VT, USA). Each concentration was repeated in three wells. The half maximal inhibitory concentration $\left(\mathrm{IC}_{50}\right)$ values were defined as the drug concentrations that reduced absorbance to $50 \%$ of control values.

\subsubsection{In vitro COX inhibition assay}

The lysates of A549 cells, which were used in COX assay, were incubated with $\mathrm{IC}_{50}$ concentrations of the compounds for 24 hours. Cells were trypsinized and collected by centrifugation at $1.000 \times g$ for $10 \mathrm{~min}$.

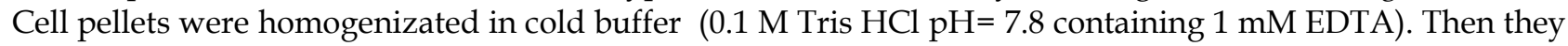
were centrifuged at $10.000 \times \mathrm{g}$ for $15 \mathrm{~min}$ at $4{ }^{\circ} \mathrm{C}$. Supernatant was removed for assay. The COX activity in A549 cell line was determined using COX activity assay kit (Cayman Chemical, Ann Arbor, MI, USA). $150 \mu \mathrm{L}$ of each sample was added to a $500 \mu \mathrm{L}$ microfuge tube then placed in boiling water for five minutes. The microfuge tubes were centrifuged at $8.000 \times \mathrm{g}$ for one minute in a microcentrifuge. Supernatants were used to 
generate the background values. $120 \mu \mathrm{L}$ assay solutions, $10 \mu \mathrm{L}$ hemin and $40 \mu \mathrm{L}$ sample and inactive samples were implemented in triplicates for sample and background wells. $110 \mu \mathrm{L}$ assay solution, $10 \mu \mathrm{L}$ hemin and 40 $\mu \mathrm{L}$ sample and $10 \mu \mathrm{L}$ DuP-697 or SC-560 were added (DuP-697 is a highly selective and irreversible COX-2 inhibitor, whereas SC-560 is a highly selective COX-1 inhibitor) for inhibitor wells. $20 \mu \mathrm{L}$ colorimetric substrat was added to all wells after the plate was shaked and incubated at $25^{\circ} \mathrm{C}$ for 5 minutes. Then, adding $20 \mu \mathrm{L}$ arachidonic acid solution started the reaction. The plate was shaked and incubated at $25^{\circ} \mathrm{C}$ for 5 minutes again. Finally, the absorbance values were recored at $540 \mathrm{~nm}$. The mean COX- 1 and COX-2 inhibition\% values of untreated A549 control cells were assumed as $0 \%$ and the inhibition rates\% of A549 cells treated with compounds $\mathbf{2 , 1 0}$ or cisplatin were calculated.

\subsubsection{Statistical analyses}

Statistical analysis were performed using Statistical Package for the Social Sciences (SPSS) for Windows 15.0. Data was shown as Mean \pm SD. Comparisons were carried out by one way ANOVA test for normally distributed continuous variables and post hoc analyses of group differences were shown by the Tukey test.

\subsection{In silico studies}

\subsubsection{Molecular docking studies}

The X-ray crystallographic structures of COX-1 and COX-2 were obtained from the PDB server (PDB IDs: 3KK6 and 5JW1, respectively) [42, 43] and optimized for docking studies in protein preparation module of Schrödinger software (Schrödinger Release 2016-2: Schrödinger, LLC, New York, NY, USA). Ligands were prepared with energy minimization using Optimized Potential Liquid Simulations (OPLS_2005) force field at physiological $\mathrm{pH}$ in ligand preparation module of Schrödinger software. In molecular docking simulations: Grid Generation and Glide/XP docking protocols were applied for the prediction of topology of compound 2 in the active sites of COX-1 and COX-2.

\subsubsection{ADME prediction}

Some physicochemical properties and the bioavailability of compounds 1-12 were calculated by QikProp module of Schrödinger software (Schrödinger Release 2016-2: QikProp, Schrödinger, LLC, New York, NY, 2016). This module computes physically significant descriptors and pharmaceutically relevant properties compared to the determined range or recommended values.

Author contributions: Concept - B.S., M.D.A.; Design - B.S., M.D.A.; Supervision - B.S., M.D.A.; Resources - B.S., M.D.A.; Materials - B.S., M.D.A., G.A.Ç.; Data Collection and/or Processing - B.S., M.D.A., G.A.Ç.; Analysis and/or Interpretation - B.S., M.D.A., G.A.Ç.; Literature Search - B.S., M.D.A.; Writing - B.S., M.D.A., G.A.Ç.; Critical Reviews - B.S., M.D.A., G.A.Ç.

Conflict of interest statement: The authors declared no conflict of interest.

\section{REFERENCES}

[1] Lemjabbar-Alaoui H, Hassan OU, Yang YW, Buchanan P. Lung cancer: Biology and treatment options. Biochim Biophys Acta. 2015; 1856: 189-210. [CrossRef]

[2] Herbst RS, Morgensztern D, Boshoff C. The biology and management of nonsmall cell lung cancer. Nature. 2018; 553: 446-454. [CrossRef]

[3] Zappa C, Mousa SA. Non-small cell lung cancer: Current treatment and future advances. Transl Lung Cancer Res. 2016; 469(3): 743-747. [CrossRef]

[4] Murphey LJ, Williams MK, Sanchez SC, Byrne LM, Csiki I, Oates JA, Johnson DH, Morrowa JD. Quantification of the major urinary metabolite of PGE2 by a liquid chromatographic/mass spectrometric assay: Determination of cyclooxygenase-specific PGE2 synthesis in healthy humans and those with lung cancer. Anal Biochem. 2004; 332: 266-275. [CrossRef]

[5] Wood SL, Pernemalm M, Crosbie, PA, Whetton AD. Molecular histology of lung cancer: From targets to treatments. Cancer Treat Rev. 2015; 41(4): 361-375. [CrossRef]

[6] Hirsch FR, Scagliotti GV, Mulshine JL, Kwon R, Curran Jr. WJ, Wu, YL, Paz-Ares L. Lung cancer: Current therapies and new targeted treatments. Lancet. 2017; 389: 299-311. [CrossRef] 
[7] Ruiz-Ceja KA, Chirino, YI. Current FDA-approved treatments for non-small cell lung cancer and potential biomarkers for its detection. Biomed Pharmacother. 2017; 90: 24-37. [CrossRef]

[8] Clària, J. Cyclooxygenase-2 biology. Curr Pharm Des. 2003; 9(27): 2177-2190. [CrossRef]

[9] Blanke C. Role of COX-2 inhibitors in cancer therapy. Cancer Invest. 2004; 22(2): 271-282. [CrossRef]

[10] Mann JR, Backlund MG, DuBois RN. Mechanisms of disease: Inflammatory mediators and cancer prevention. Nat Clin Pract Oncol. 2005; 2(4): 202-210. [CrossRef]

[11] Jeon YT, Song YS. Cyclooxygenases in cancer: chemoprevention and sensitization to conventional therapies. Mini Rev Med Chem. 2006; 6(7): 827-833. [CrossRef]

[12] Goradel NH, Najafi M, Salehi E, Farhood B, Mortezaee K. Cyclooxygenase-2 in cancer: A review. J Cell Physiol. 2019; 234(5): 5683-5699. [CrossRef]

[13] Hida T, Yatabe Y, Achiwa H, Muramatsu H, Kozaki K, Nakamura S, Ogawa M, Mitsudomi T, Sugiura T, Takahashi $\mathrm{T}$. Increased expression of cyclooxygenase 2 occurs frequently in human lung cancers, specifically in adenocarcinomas. Cancer Res. 1998; 58(17): 3761-3764.

[14] Khuri FR, Wu H, Lee JJ, Kemp BL, Lotan R, Lippman SM, Feng L, Hong WK, Xu XC. Cyclooxygenase-2 overexpression is a marker of poor prognosis in stage I non-small cell lung cancer. Clin Cancer Res. 2001; 7(4): 861867.

[15] Altorki NK, Port JL, Zhang F, Golijanin D, Thaler HT, Duffield-Lillico AJ, Subbaramaiah K, Dannenberg, AJ. Chemotherapy induces the expression of cyclooxygenase-2 in non-small cell lung cancer. Clin Cancer Res. 2005; 11(11): 4191-4197. [CrossRef]

[16] Yuan A, Yu CJ, Shun CT, Luh K-T, Kuo S-H, Lee Y-C, Yang P-C. Total cyclooxygenase-2 mRNA levels correlate with vascular endothelial growth factor mRNA levels, tumor angiogenesis and prognosis in non-small cell lung cancer patients. Int J Cancer. 2005; 115(4): 545-555. [CrossRef]

[17] Lim ES, Rhee YH, Park MK, Shim B-S, Ahn K-S, Kang H, Yoo H-S, Kim S-H. DMNQ S-64 induces apoptosis via caspase activation and cyclooxygenase-2 inhibition in human nonsmall lung cancer cells. Ann N Y Acad Sci. 2007; 1095: 7-18. [CrossRef]

[18] Greene ER, Huang S, Serhan CN, Panigrahy D. Regulation of inflammation in cancer by eicosanoids. Prostaglandins Other Lipid Mediat. 2011; 96(1-4): 27-36. [CrossRef]

[19] Mattsson JS, Bergman B, Grinberg M, Edlund K, Marincevic M, Jirström K, Pontén F, Hengstler JG, Rahnenführer J, Karlsson MG, Karlsson C, Helenius G, Botling J, Micke P, Gulyas M. Prognostic impact of COX-2 in non-small cell lung cancer: a comprehensive compartment-specific evaluation of tumor and stromal cell expression. Cancer Lett. 2015; 356(2 Pt B): 837-845. [CrossRef]

[20] Dai P, Li J, Ma XP, Huang J, Meng JJ, Gong P. Efficacy and safety of COX-2 inhibitors for advanced non-small-cell lung cancer with chemotherapy: A meta-analysis. Onco Targets Ther. 2018; 11: 721-730. [CrossRef]

[21] Zhang P, He D, Song E, Jiang M, Song Y. Celecoxib enhances the sensitivity of non-small-cell lung cancer cells to radiation-induced apoptosis through downregulation of the Akt/mTOR signaling pathway and COX-2 expression [published correction appears in PLoS One. 2019; 14(10):e0224843]. PLoS One. 2019; 14(10): e0223760. [CrossRef]

[22] Yu T, Lao X, Zheng H. Influencing COX-2 activity by COX related pathways in inflammation and cancer. Mini Rev Med Chem. 2016; 16(15): 1230-1243. [CrossRef]

[23] Pannunzio A, Coluccia M. Cyclooxygenase-1 (COX-1) and COX-1 inhibitors in cancer: A review of oncology and medicinal chemistry literature. Pharmaceuticals (Basel). 2018; 11(4): 101. [CrossRef]

[24] Vitale P, Scilimati A, Perrone MG. Update on SAR studies toward new COX-1 selective inhibitors. Curr Med Chem. 2015; 22(37): 4271-4292. [CrossRef]

[25] Rocca B, Morosetti R, Habib A, Maggiano N, Zassadowski F, Ciabattoni G, Chomienne C, Papp B, Ranelletti FO. Cyclooxygenase-1, but not -2 , is upregulated in NB4 leukemic cells and human primary promyelocytic blasts during differentiation. Leukemia. 2004; 18(8): 1373-1379. [CrossRef]

[26] Saha R, Tanwar O, Marella A, Alam MM, Akhter M. Recent updates on biological activities of oxadiazoles. Mini Rev Med Chem. 2013; 13(7): 1027-1046. [CrossRef]

[27] Bajaj S, Asati V, Singh J, Roy PP. 1,3,4-Oxadiazoles: An emerging scaffold to target growth factors, enzymes and kinases as anticancer agents. Eur J Med Chem. 2015; 97: 124-141. [CrossRef] 
[28] Miller K, Moul JW, Gleave M, Fizazi K, Nelson JB, Morris T, Nathan FE, McIntosh S, Pemberton K, Higano CS. Phase III, randomized, placebo-controlled study of once-daily oral zibotentan (ZD4054) in patients with non-metastatic castration-resistant prostate cancer. Prostate Cancer Prostatic Dis. 2013; 16(2): 187-192. [CrossRef]

[29] Fargualy AM, Habib NS, Ismail KA, Hassan AM, Sarg MT. Synthesis, biological evaluation and molecular docking studies of some pyrimidine derivatives. Eur J Med Chem. 2013; 66: 276-295. [CrossRef]

[30] Kaur R, Kaur P, Sharma S, Singh G., Mehndiratta S, Bedi PMS, Nepali K. Anti-cancer pyrimidines in diverse scaffolds: a review of patent literature. Recent Pat Anticancer Drug Discov. 2015; 10(1): 23-71. [CrossRef]

[31] Myungho J, Joo Bin K, Hyeonho J, Younho L, Hua L, Sein K, Hyun JS, Juhee K, Hwan K, Hyunkyung K, Kyung-Ah S, Jung Beom S, Nam Doo K, Jiyoon S, Sun-Hwa L. Prepn. of 2,4,5-substituted pyrimidine derivs. for preventing or treating cancer. PCT Int. Appl. (2019), WO 2019177375 A1 20190919.

[32] Yildirim M, Yildirim A, Mutlu, E. Novel thiazolo[3,2-c] pyrimidine based anticancer compounds. PCT Int. Appl. (2019), WO 2019240681 A2 20191219.

[33] Bedi S, Khan SA, AbuKhader MM, Alam P, Siddiqui NA, Husain A. A comprehensive review on Brigatinib - A wonder drug for targeted cancer therapy in non-small cell lung cancer. Saudi Pharm J. 2018; 26(6): 755-763. [CrossRef]

[34] Zhang Y, Lv H, Luo L, Xu Y, Pan Y, Wang Y, Lin H, Xiong J, Guo P, Zhang J, Li X, Ye F. Design, synthesis and pharmacological evaluation of N4,N6-disubstituted pyrimidine-4,6-diamine derivatives as potent EGFR inhibitors in non-small cell lung cancer. Eur J Med Chem. 2018; 157: 1300-1325. [CrossRef]

[35] De la Torre BG, Albericio F. The pharmaceutical industry in 2017. An analysis of FDA drug approvals from the perspective of molecules. Molecules. 2018; 23: 533. [CrossRef]

[36] Dannhardt G, Kiefer W. Cyclooxygenase inhibitors--current status and future prospects. Eur J Med Chem. 2001; 36(2): 109-126. [CrossRef]

[37] Orjales A, Mosquera R, López B, Olivera R, Labeaga L, Núñez MT. Novel 2-(4-methylsulfonylphenyl)pyrimidine derivatives as highly potent and specific COX-2 inhibitors. Bioorg Med Chem. 2008; 16(5): 2183-2199. [CrossRef]

[38] Sondhi SM, Singhal N, Johar M, Reddy BS, Lown JW. Heterocyclic compounds as inflammation inhibitors. Curr Med Chem. 2002; 9(10): 1045-1074. [CrossRef]

[39] Güngör EM, Altıntop MD, Sever B, Akalın Çiftçi G. Design, synthesis, in vitro and in silico evaluation of new hydrazone-based antitumor agents as potent Akt inhibitors. Lett Drug Des Discov. 2020. [CrossRef]

[40] Altıntop MD, Sever B, Akalın Çiftçi G, Turan-Zitouni G, Kaplancıklı ZA, Özdemir A. Design, synthesis, in vitro and in silico evaluation of a new series of oxadiazole-based anticancer agents as potential Akt and FAK inhibitors. Eur J Med Chem. 2018; 155: 905-924. [CrossRef]

[41] Mosmann T. Rapid colorimetric assay for cellular growth and survival: application to proliferation and cytotoxicity assays. J Immunol Methods 1983; 65: 55-63. [CrossRef]

[42] Rimon G., Sidhu RS, Lauver DA, Lee JY, Sharma NP, Yuan C, Frieler RA, Trievel RC, Lucchesi BR, Smith WL. Coxibs interfere with the action of aspirin by binding tightly to one monomer of cyclooxygenase-1. Proc Natl Acad Sci USA. 2010; 107: 28-33 [CrossRef]

[43] Dong L, Yuan C, Orlando BJ, Malkowski MG, Smith WL. Fatty acid binding to the allosteric subunit of cyclooxygenase-2 relieves a tonic inhibition of the catalytic subunit. J Biol Chem. 2016; 291: 25641-25655. [CrossRef] 